$(6 / 6 / 2007,14 \mathrm{~h} 19)$

\title{
Moyennes de certaines fonctions multiplicatives sur les entiers friables, 2
}

\author{
Guillaume Hanrot, Gérald Tenenbaum \& Jie Wu
}

\begin{abstract}
We derive new, very precise estimates for averages of arithmetic functions over friable integers from analytic information on their associated Dirichlet series. These yield significant improvements upon available results in classical cases, in particular concerning the effective expansion of "abstract" main terms of de Bruijn type. These results also permit new applications, linked to the solubility of polynomial equations.
\end{abstract}

AMS Subject classification. 11N37, 11N25, 11M41, 11C08.

Keywords. Friable integers, averages of multiplicative functions, polynomials, Dedekind zeta functions, solubility of polynomial equations.

\section{Sommaire}

1 Introduction . . . . . . . . . . . 1

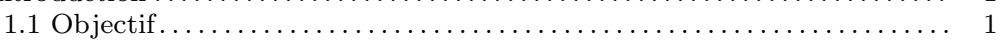

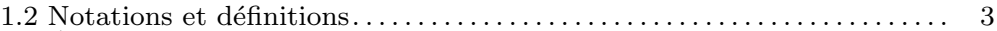

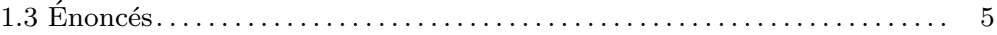

1.4 Description sommaire d'une généralisation $\ldots \ldots \ldots \ldots \ldots \ldots \ldots \ldots \ldots . . \ldots \ldots$

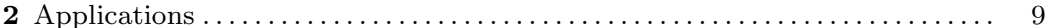

2.1 Entiers représentables comme somme de deux carrés .......... 9

2.2 Répartition des valeurs de la fonction d'Euler . . . . . . . . . . . . 10

2.3 Nombre de solutions de congruences polynomiales modulo

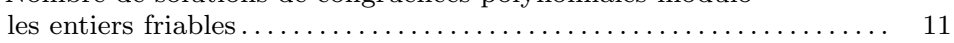

2.4 Solubilité de congruences polynomiales modulo

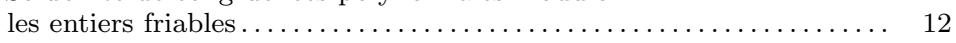

3 Développement asymptotique du terme principal $\ldots \ldots \ldots \ldots \ldots \ldots \ldots \ldots . \ldots 13$

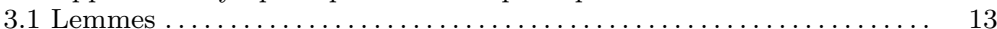

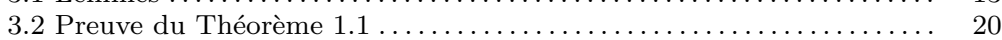

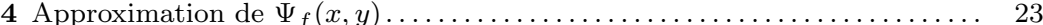

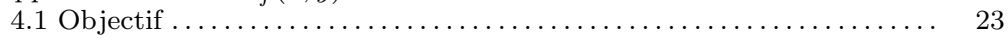

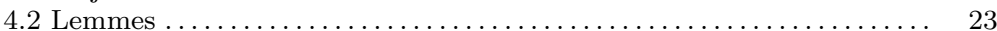

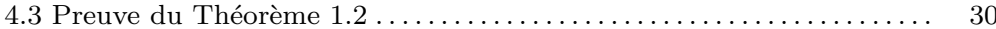

5 Fonctions zêtas de Dedekind généralisées $\ldots \ldots \ldots \ldots \ldots \ldots \ldots \ldots \ldots \ldots \ldots \ldots \ldots$

\section{Introduction}

\subsection{Objectif}

Désignons par $P(n)$ le plus grand facteur premier d'un entier naturel $n>1$ et convenons que $P(1)=1$. Dans la première partie de cet article [20], les deux derniers auteurs ont obtenu des évaluations pour la fonction sommatoire

$$
\Psi_{f}(x, y):=\sum_{n \in S(x, y)} f(n)
$$


de certaines fonctions arithmétiques multiplicatives positives ou nulles sur l'ensemble

$$
S(x, y):=\{n \leqslant x: P(n) \leqslant y\}
$$

des entiers $y$-friables n'excédant pas $x$.

Alors que les hypothèses considérées dans [20] consistent essentiellement à supposer que les nombres $f(p)$ possèdent, lorsque $p$ décrit la suite des nombres premiers, une valeur moyenne strictement positive, il est également signalé dans ce travail qu'une information supplémentaire de type prolongement analytique pour la série de Dirichlet $\mathcal{F}(s)$ associée à $f$ peut être exploitée en vue d'améliorer significativement la précision des formules asymptotiques obtenues pour la quantité (1·1).

Notons $\tau_{\kappa}(n)$ le coefficient d'indice $n$ de la série de Dirichlet $\zeta(s)^{\kappa}$ où $\zeta$ désigne la fonction zêta de Riemann. Des exemples de la situation favorable évoquée ci-dessus ont été traités, par la méthode du col, dans [10] et [16] lorsque $\kappa=1$ et dans [14] pour tout $\kappa>0$.

Dans [20] (théorème 2.5), Tenenbaum et Wu ont précisé, sans démonstration, le résultat obtenu dans le cas où $f$ est la fonction indicatrice des entiers représentables comme somme de deux carrés. La série de Dirichlet impliquée est alors comparable à $\zeta(s)^{1 / 2}$ mais, contrairement aux exemples précédents, la suite des $f(p)$ n'est pas constante. Cela illustre la souplesse de la méthode, qui peut être adaptée sans altération majeure au cas d'une série de Dirichlet de la forme

$$
\mathcal{F}(s)=\zeta(s)^{\kappa} G(s)
$$

où $\kappa$ est un nombre réel positif et $G(s)$ est une série de Dirichlet présentant de bonnes propriétés de prolongement analytique.

La même approche est pertinente, sans modification profonde, pour une série de Dirichlet analytiquement proche d'une fonction zêta de Dedekind, comme dans le récent travail de Scourfield [11], où, toutefois, la méthode employée ne fournit pas la totalité les renseignements attendus. Nous verrons au paragraphe 5 que la technique décrite dans [20] permet en fait de réduire les hypothèses concernant $\mathcal{F}(s)$ au cas d'une série analytiquement proche d'un produit eulérien dont le terme général est bien approché par une expression du type

$$
1+\mathcal{J}\left(\varrho_{F}(p)\right) / p^{s}
$$

où $F \in \mathbb{Z}[X], \varrho_{F}(p)$ est le nombre des racines de $F$ dans $\mathbb{Z} / p \mathbb{Z}$ et $\mathcal{J}$ est une fonction arbitraire de $\mathbb{Z}$ dans $\mathbb{R}^{+}$.

Par souci de clarification et à fins de référence ultérieure, nous proposons dans ce travail un énoncé général recouvrant essentiellement l'ensemble des cas connus relevant de la méthode du col. Notre résultat principal est le Théorème 1.2 infra. Il englobe stricto sensu le cas de fonctions non multiplicatives. Cet énoncé pourrait d'ailleurs être encore étendu au prix de certaines complications techniques que nous avons préféré éviter. 


\subsection{Notations et définitions}

Nous notons

$$
M(x ; f):=\sum_{1 \leqslant n \leqslant x} f(n) \quad(x \geqslant 0)
$$

la fonction sommatoire d'une fonction arithmétique $f$.

La lettre $s$ désignant un nombre complexe, nous définissons implicitement les nombres réels $\sigma$ et $\tau$ par $s=\sigma+i \tau$.

Pour $\beta \in] 0,3 / 5[$, nous posons

$$
L_{\beta}(y):=\mathrm{e}^{(\log y)^{\beta}} \quad(y \geqslant 1) .
$$

Nous notons $\zeta_{\mathbb{K}}$ la fonction zêta de Dedekind d'un corps de nombres $\mathbb{K}$.

Pour $\kappa>0$, nous désignons par $\mathcal{D}_{\kappa}$ la classe des séries de Dirichlet $s \mapsto Z(s)$ représentables sous la forme

$$
Z(s)=\prod_{1 \leqslant j \leqslant r} \zeta_{\mathbb{K}_{j}}(s)^{\kappa_{j}}
$$

où les $\mathbb{K}_{j}$ sont des corps de nombres arbitraires et les $\kappa_{j}$ sont des nombres réels non nuls tels que

$$
\sum_{1 \leqslant j \leqslant r} \kappa_{j}=\kappa
$$

Nous posons alors

$$
w_{Z}:=\sum_{1 \leqslant j \leqslant r} \kappa_{j}\left[\mathbb{K}_{j}: \mathbb{Q}\right]
$$

où $\left[\mathbb{K}_{j}: \mathbb{Q}\right]$ désigne la dimension de $\mathbb{K}_{j}$ comme $\mathbb{Q}$-espace vectoriel. Lorsque $\kappa \in \mathbb{N}^{*}$, nous notons $\mathcal{D}_{\kappa}^{*}$ la sous-classe de $\mathcal{D}_{\kappa}$ constituée des séries $Z(s)$ possédant une décomposition de type $(1 \cdot 3)$ dans laquelle chaque $\kappa_{j}$ est un entier naturel positif.

Ensuite, nous introduisons, pour $\beta>0, c>0, \delta>0, \beta+\delta<3 / 5$, la classe $\mathcal{E}_{\kappa}(\beta, c, \delta)$ des séries de Dirichlet $\mathcal{F}(s)$ convergentes pour $\sigma>1$ et possédant dans ce demi-plan une décomposition de la forme

$$
\mathcal{F}(s)=Z(s) G(s)
$$

où $Z \in \mathcal{D}_{\kappa}$ et $G(s)=\sum_{n \geqslant 1} g(n) / n^{s}$ est une série de Dirichlet prolongeable holomorphiquement au domaine

$$
\sigma>1-c /\left\{1+\log ^{+}|\tau|\right\}^{(1-\beta-\delta) /(\beta+\delta)}
$$

où elle vérifie les conditions

$$
G(1) \neq 0, \quad G(s) \ll\{1+|\tau|\}^{1-\delta} .
$$


Ici et dans la suite, nous posons traditionnellement $\log ^{+} x:=\max (\log x, 0)(x>0)$.

Similairement, lorsque $\kappa \in \mathbb{N}^{*}$, nous désignons par $\mathcal{E}_{\kappa}^{*}(\delta)$ la classe des séries de Dirichlet $\mathcal{F}(s)$ possédant dans le demi-plan $\sigma>1$ une décomposition de la forme (1.5) où $Z \in \mathcal{D}_{\kappa}^{*}$ et $G$ est prolongeable, sur le demi-plan $\sigma \geqslant 1-\delta$, en une fonction holomorphe vérifiant $(1 \cdot 7)$.

Nous désignons par $\mathcal{H}\left(\kappa, \kappa_{0} ; \beta, c, \delta\right)$ - resp. $\mathcal{H}^{*}\left(\kappa, \kappa_{0} ; \delta\right)$ - la classe des fonctions arithmétiques $f$ dont la série de Dirichlet associée

$$
\mathcal{F}(s):=\sum_{n \geqslant 1} f(n) / n^{s}
$$

appartient à $\mathcal{E}_{\kappa}(\beta, c, \delta)$ - resp. à $\mathcal{E}_{\kappa}^{*}(\delta)$ - et possède une série majorante $\mathcal{F}^{*}(s)=Z^{*}(s) G^{*}(s)$ dans $\mathcal{E}_{\kappa_{0}}(\beta, c, \delta)$ - resp. dans $\mathcal{E}_{\kappa_{0}}^{*}(\delta)$. Lorsque $f$ appartient à $\mathcal{E}_{\kappa}(\beta, c, \delta)$ ou à $\mathcal{E}_{\kappa_{0}}^{*}(\delta)$, nous posons, avec la notation (1.4)

$$
v_{f}:=\max \left\{w_{Z}, w_{Z^{*}}\right\} .
$$

Enfin, étant donnée une famille de fonctions arithmétiques $\left\{g_{y}\right\}_{y \geqslant 2}$ telle que $\lim _{y \rightarrow \infty} g_{y}(n)=g(n)(n \geqslant 1)$, nous notons $\mathcal{H}_{+}(\kappa ; \beta, c, \delta)$ et $\mathcal{H}_{+}^{*}(\kappa ; \beta, c, \delta)$ les sousclasses respectives de $\mathcal{H}(\kappa, \kappa ; \beta, c, \delta)$ et $\mathcal{H}^{*}(\kappa, \kappa ; \delta)$ correspondant aux fonctions $f$ positives ou nulles satisfaisant en outre à la condition

$$
G(s ; y):=\sum_{n \geqslant 1} \frac{g_{y}(n)}{n^{s}}=G(s)+O\left(\frac{1}{L_{\beta+\delta}(y)}\right)
$$

uniformément pour

$$
y \geqslant 2, \quad \sigma>1-c /(\log y)^{1-\beta-\delta}, \quad|\tau| \leqslant L_{\beta+\delta}(y) .
$$

Nous aurons plusieurs fois l'occasion d'utiliser la relation d'inclusion

$$
\mathcal{H}_{+}(\kappa ; \beta, c, \delta) \subset \mathcal{H}_{+}(\kappa ; \beta+\delta / 2, c, \delta / 2),
$$

valable pour toutes les valeurs des paramètres considérées, lorsque la constante $c$ est assez petite, ce que nous supposons dans toute la suite de ce travail.

L'introduction des fonctions $g_{y}$, qui peut, au premier abord, sembler surprenante, permet d'étendre considérablement le champ des applications. Notons $t_{\kappa}$ la fonction arithmétique associée à la série de Dirichlet (1.3) et $t_{-\kappa}$ son inverse de convolution. Nous avons $f=t_{\kappa} * g$. Posons alors, en notant $S(y):=\left\{n \in \mathbb{N}^{*}: P(n) \leqslant y\right\}$,

$$
f_{y}:=\left(\mathbf{1}_{S(y)} t_{\kappa}\right) * g_{y} .
$$

Pour le choix standard

$$
g_{y}:=\mathbf{1}_{S(y)} g,
$$


nous avons

$$
M\left(x ; f_{y}\right)=\Psi_{f}(x, y) .
$$

Cependant, d'autres spécialisations, comme celle décrite au paragraphe 2.2, permettent d'englober dans notre étude des fonctions sommatoires d'intérêt arithmétique significatif. Supposons par exemple que l'on a $f(n)=\sum_{h(m)=n} r(m)$, où $h$ et $r$ sont des fonctions arithmétiques convenables vérifiant $h\left(\mathbb{N}^{*}\right) \subset \mathbb{N}^{*}$. Ainsi

$$
\mathcal{F}(s)=\sum_{n \geqslant 1} \frac{f(n)}{n^{s}}=\sum_{m \geqslant 1} \frac{r(m)}{h(m)^{s}}
$$

et $g(n)=\sum_{h(d) m=n} r(d) t_{-\kappa}(m)$. Pour le choix

$$
g_{y}(n)=\sum_{\substack{P(d m) \leqslant y \\ h(d) m=n}} r(d) t_{-\kappa}(m)
$$

nous obtenons

$$
M\left(x ; f_{y}\right)=\sum_{\substack{h(m) \leqslant x \\ P(m) \leqslant y}} r(m)
$$

\section{3. Énoncés}

Soient $\beta, c, \delta, \kappa, \kappa_{0}$, des nombres réels positifs tels que $\beta+\delta<3 / 5$ et $f$ une fonction arithmétique de $\mathcal{H}\left(\kappa, \kappa_{0} ; \beta, c, \delta\right)$. La méthode de Selberg-Delange décrite aux chapitres II.5 et II.6 de [16] permet d'évaluer la fonction sommatoire de $f$. Introduisant le développement de Taylor

$$
\frac{s^{\kappa} \mathcal{F}(s+1)}{s+1}=\sum_{j \geqslant 0} a_{j}(f) s^{j}
$$

convergent pour $|s| \leqslant \frac{1}{2} c$, on obtient ainsi, pour tout entier naturel $J$,

$$
M(x ; f)=x(\log x)^{\kappa-1}\left\{\sum_{0 \leqslant j \leqslant J} \frac{a_{j}(f)}{\Gamma(\kappa-j)(\log x)^{j}}+O\left(\mathcal{R}_{J}(x)\right)\right\},
$$

où l'on a posé, pour des constantes convenables $c_{1}>0, c_{2}>0$,

$$
\mathcal{R}_{J}(x):=\frac{1}{L_{\beta+\delta}(x)^{c_{1}}}+\left(\frac{c_{2} J+1}{\log x}\right)^{J+1}
$$

et où la constante implicite est indépendante de $J$. Si, de plus, $\kappa \in \mathbb{N}^{*}$ et $f \in \mathcal{H}^{*}\left(\kappa, \kappa_{0} ; \delta\right)$, alors $a_{j}(f) / \Gamma(\kappa-j)=0$ pour $j \geqslant \kappa$ et l'on peut choisir

$$
\mathcal{R}_{\kappa}(x)=1 / x^{\sigma}
$$

pour tout $\sigma$ vérifiant, par exemple, $0<\sigma<\delta / \max \left\{v_{f}, 2\right\}$. Cette dernière borne est d'ailleurs susceptible d'améliorations diverses qu'il nous entraînerait trop loin de notre sujet de discuter ici. 
Comme dans [20], nous notons $z_{\kappa}(u)$ la solution continue sur ]0, $\infty$ [ de l'équation différentielle aux différences

$$
\begin{cases}z_{\kappa}(u)=0 & \text { si } u<0 \\ z_{\kappa}(u)=1 & \text { si } 0 \leqslant u \leqslant 1 \\ u z_{\kappa}^{\prime}(u)=-\kappa z_{\kappa}(u-1) & \text { si } u>1\end{cases}
$$

Ainsi qu'il a été mentionné dans [20], la fonction $z_{\kappa}$ est liée à la puissance de convolution fractionnaire $\varrho_{\kappa}$ de la fonction de Dickman $\varrho:=\varrho_{1}=z_{1}$ par la relation

$$
\int_{0}^{u} \varrho_{\kappa}(v) \mathrm{d} v=\frac{1}{\Gamma(\kappa)} \int_{0}^{u} v^{\kappa-1} z_{\kappa}(u-v) \mathrm{d} v \quad(u>0) .
$$

La transformée de Laplace de $\varrho_{\kappa}$ est donnée par

$$
\widehat{\varrho}_{\kappa}(s):=\int_{0}^{\infty} \mathrm{e}^{-u s} \varrho_{\kappa}(u) \mathrm{d} u=\widehat{\varrho}(s)^{\kappa}=\exp \left\{\gamma \kappa+\kappa \int_{0}^{-s} \frac{\mathrm{e}^{v}-1}{v} \mathrm{~d} v\right\},
$$

où $\gamma$ désigne la constante d'Euler. Pour d'autres informations concernant $\varrho$ et $\varrho_{\kappa}$, on pourra notamment consulter [16] (chap. III.5) et [13].

Notre approximation pour $M\left(x ; f_{y}\right)$ est l'expression intégrale de Stieltjes

$$
\Lambda_{f}(x, y):=x \int_{\mathbb{R}} z_{\kappa}(u-v) \mathrm{d}\left(\frac{M\left(y^{v} ; f\right)}{y^{v}}\right) .
$$

Ici et dans la suite, nous posons

$$
u:=\frac{\log x}{\log y} \quad(x \geqslant 2, y \geqslant 2) .
$$

Ce type de terme principal peut se révéler remarquablement efficace dans certaines applications, notamment lorsqu'il doit être réinséré dans une sommation, comme par exemple dans les problèmes de la valeur moyenne du logarithme du plus grand ou du plus petit facteur premier d'un entier, traités respectivement à l'exercice III.5.3 de [16] (résolu dans [19]) et au corollaire 4 de [17]. Il est cependant légitime de donner d'emblée une approximation plus concrète de la quantité $(1 \cdot 21)$.

Notons $\langle x\rangle$ la partie fractionnaire d'un nombre réel $x$ et posons

$$
\vartheta:=\langle\kappa\rangle, \quad m:=\kappa-\vartheta \in \mathbb{N} .
$$

Sous certaines conditions de croissance de $y$ en fonction de $x$, nous sommes en mesure d'établir une formule asymptotique pour $\Lambda_{f}(x, y)$ lorsque $f \in \mathcal{H}\left(\kappa, \kappa_{0} ; \beta, c, \delta\right)$ et un développement asymptotique lorsque le paramètre $u:=(\log x) / \log y$ n'est pas trop proche, par valeurs supérieures, d'un entier borné. Notant $\log _{k}$ la $k$-ième itérée de la fonction logarithme, nous posons

$$
\varepsilon_{J, y}:=\left\{(J+1) \log _{2} y\right\}^{1 / \beta} / \log y \quad(y \geqslant 2) .
$$


Théorème 1.1. Soient $\beta, c, \delta, \kappa, \kappa_{0}$, des nombres réels positifs tels que $\beta+\delta<3 / 5$, et $f \in \mathcal{H}\left(\kappa, \kappa_{0} ; \beta, c, \delta\right)$. Nous avons

$$
\Lambda_{f}(x, y)=a_{0}(f) x(\log y)^{\kappa-1} \varrho_{\kappa}(u)\left\{1+O\left(\frac{1}{(\log y)^{\kappa}}+\frac{\log (u+1)}{\log y}\right)\right\}
$$

uniformément, sous l'hypothèse

$$
x \geqslant 3, \quad \exp \left\{(\log x)^{1-\beta}\right\} \leqslant y \leqslant x .
$$

De plus, pour chaque entier naturel $J$ et sous la condition supplémentaire

$\left(\mathcal{G}_{J}(\kappa)\right)$

$$
0<u<J+2-\kappa \Rightarrow\langle u\rangle>\varepsilon_{J, y}
$$

nous avons

$$
\Lambda_{f}(x, y)=x(\log y)^{\kappa-1}\left\{\sum_{0 \leqslant j \leqslant J} \frac{a_{j}(f) \varrho_{\kappa}^{(j)}(u)}{(\log y)^{j}}+O\left(\varrho_{\kappa}(u)\left\{\frac{\log (u+1)}{\log y}\right\}^{J+1}\right)\right\} .
$$

Sous l'hypothèse supplémentaire $\kappa \in \mathbb{N}^{*}, \kappa_{0} \in \mathbb{N}^{*}, f \in \mathcal{H}^{*}\left(\kappa, \kappa_{0} ; \delta\right)$, les assertions précédentes sont valables pour le choix $\varepsilon_{J, y}:=B(J+1) \log _{2} y / \log y$, où $B$ est une constante assez grande, et en remplaçant la condition $\left(G_{\beta}\right)$ par

$$
(\log x)^{1+\varepsilon} \leqslant y \leqslant x .
$$

Nous pouvons à présent expliciter notre résultat principal.

Théorème 1.2. Soient $\beta, c, \delta, \kappa$, des nombres réels positifs tels que $\beta+\delta<3 / 5$, et $f \in \mathcal{H}_{+}(\kappa ; \beta, c, \delta)$. La formule asymptotique

$$
M\left(x ; f_{y}\right)=\left\{1+O\left(\frac{1}{L_{\beta}(y)}\right)\right\} \Lambda_{f}(x, y)
$$

a lieu uniformément dans le domaine $\left(G_{\beta}\right)$.

Si, de plus, $\kappa \in \mathbb{N}^{*}$ et $f \in \mathcal{H}_{+}^{*}(\kappa ; \beta, c, \delta)$ la formule (1.26) a lieu uniformément dans le domaine

$$
x \geqslant 3, \quad \exp \left\{\left(\log _{2} x\right)^{1 / \beta}\right\} \leqslant y \leqslant x .
$$

Remarques. (i) Il résulte d'un cas particulier des résultats de [20] que l'on a, dans le domaine $\left(H_{\beta}\right)$,

$$
\Psi_{f}(x, y)=a_{0}(f) x \varrho_{\kappa}(u)(\log y)^{\kappa-1}\left\{1+O\left(\frac{1}{(\log y)^{\kappa}}+\frac{\log (u+1)}{\log y}\right)\right\}
$$


lorsque $f$ est multiplicative, positive ou nulle, et vérifie

$$
\sum_{p \leqslant z} f(p) \log p=\kappa z+O\left(z / L_{\beta}(z)\right) \quad(z \geqslant 2) .
$$

La conjonction de (1.25) et (1.26) établit donc, au prix d'une relative restriction du domaine de validité, une formule asymptotique considérablement plus précise. On retrouve de plus le domaine de validité $\left(H_{\beta}\right)$ lorsque $\kappa \in \mathbb{N}^{*}$ et $f \in \mathcal{H}_{+}^{*}(\kappa ; \beta, c, \delta)$.

(ii) Le cas le plus simple du Théorème 1.2 , soit $\mathbb{K}=\mathbb{Q}, \kappa=r=1, G_{\kappa}(s)=1$, correspond au théorème de Saias [10].

(iii) Lorsque $\kappa=r=1, G(s)=1$, nous obtenons une amélioration significative des théorèmes 1.1 et 1.3 de [11].

(iv) La formule (1.26) généralise le résultat de Smati et $\mathrm{Wu}$ [12], qui correspond au choix $r=\mathbf{1}, t_{-\kappa}=\mu$ et $h=\varphi$ dans (1.15).

\subsection{Description sommaire d'une généralisation}

La méthode développée dans ce travail permet, sans difficulté nouvelle, de traiter un cas plus général, que nous nous proposons de décrire succinctement dans ce paragraphe.

Soient $r \in \mathbb{N}^{*},\left\{\alpha_{j}\right\}_{j=1}^{r} \in \mathbb{N}^{* r}, \kappa>0$. Pour chaque $j$ de $[1, r]$, donnons-nous une suite strictement croissante $\left\{\nu_{j, i}\right\}_{i=1}^{\alpha_{j}}$ d'entiers positifs et une suite $\left\{\kappa_{j, i}\right\}_{i=1}^{\alpha_{j}}$ de nombres réels non nuls telle que

$$
\sum_{1 \leqslant j \leqslant r} \sum_{1 \leqslant i \leqslant \alpha_{j}} \kappa_{j, i}=\kappa
$$

Nous pouvons alors étendre la classe $\mathcal{D}_{\kappa}$ à l'ensemble $\widetilde{\mathcal{D}}_{\kappa}$ des séries de Dirichlet de la forme

$$
Z(s):=\prod_{1 \leqslant j \leqslant r} \prod_{1 \leqslant i \leqslant \alpha_{j}} \zeta_{\mathbb{K}_{j}}\left(1+\nu_{j, i}(s-1)\right)^{\kappa_{j, i}}
$$

où les $\mathbb{K}_{j}$ sont des corps de nombres arbitraires.

Pour $\beta>0, c>0, \delta>0$, et $\beta+\delta<3 / 5, \kappa>0, \kappa_{0}>0$, nous introduisons alors les extensions correspondantes $\widetilde{\mathcal{D}}_{\kappa}^{*}$ (où tous les $\kappa_{j, i}$ sont entiers), $\widetilde{\mathcal{E}}_{\kappa}(\beta, c, \delta), \widetilde{\mathcal{E}}_{\kappa}^{*}(\delta)$, $\widetilde{\mathcal{H}}\left(\kappa, \kappa_{0} ; \beta, c, \delta\right), \widetilde{\mathcal{H}}\left(\kappa, \kappa_{0} ; \delta\right), \widetilde{\mathcal{H}}_{+}(\kappa ; \beta, c, \delta), \widetilde{\mathcal{H}}_{+}^{*}(\kappa ; \beta, c, \delta)$.

Posons $\varrho_{\kappa, \nu}(u):=\varrho_{\kappa}(u / \nu)$ et, notant

$$
\begin{aligned}
& \boldsymbol{\nu}:=\left(\nu_{1,1}, \ldots, \nu_{1, \alpha_{1}}, \ldots, \nu_{r, 1}, \ldots, \nu_{r, \alpha_{r}}\right), \\
& \boldsymbol{\kappa}:=\left(\kappa_{1,1}, \ldots, \kappa_{1, \alpha_{1}}, \ldots, \kappa_{r, 1}, \ldots, \kappa_{r, \alpha_{r}}\right),
\end{aligned}
$$

introduisons le produit de convolution

$$
\varrho(u ; \boldsymbol{\kappa}, \boldsymbol{\nu}):=\underset{\substack{1 \leqslant j \leqslant r \\ 1 \leqslant i \leqslant \alpha_{j}}}{*} \varrho_{\kappa_{j, i}, \nu_{j, i}}(u) .
$$


Si $f$ est une fonction arithmétique dont la série de Dirichlet $\mathcal{F}(s)=Z(s) G(s)$ appartient à $\widetilde{\mathcal{E}}_{\kappa}(\beta, c, \delta)$, les démonstrations des Théorèmes 1.1 et 1.2 peuvent être étendues directement. Nous obtenons ainsi que, si $f \in \widetilde{\mathcal{H}}_{+}(\kappa ; \beta, c, \delta)$, on a pour tout entier $J \geqslant 0$,

$$
\frac{M\left(x ; f_{y}\right)}{x(\log y)^{\kappa-1}}=\sum_{0 \leqslant j \leqslant J} \frac{a_{j}(f) \varrho^{(j)}(u ; \boldsymbol{\kappa}, \boldsymbol{\nu})}{(\log y)^{j}}+O\left(\varrho(u ; \boldsymbol{\kappa}, \boldsymbol{\nu})\left\{\frac{\log (u+1)}{\log y}\right\}^{J+1}\right)
$$

uniformément sous les conditions $\left(G_{\beta}\right)$ et $\mathcal{G}_{J}(\kappa)$. Si, de plus, $\kappa \in \mathbb{N}^{*}$ et $f \in$ $\widetilde{\mathcal{H}}_{+}^{*}(\kappa ; \beta, c, \delta)$, on peut remplacer $\left(G_{\beta}\right)$ par $\left(H_{\beta}\right)$ et choisir $\varepsilon_{J, y}$ comme indiqué dans la seconde partie de l'énoncé du Théorème 1.1.

Le point essentiel pour parvenir à cette généralisation consiste à remarquer que, si $f$ est une fonction arithmétique dont la série de Dirichlet $\mathcal{F}(s)=Z(s) G(s)$ appartient à $\widetilde{\mathcal{E}}_{\kappa}(\beta, c, \delta)$, la relation $(4 \cdot 2)$ infra appliquée avec $\mathbb{K}=\mathbb{K}_{j}(1 \leqslant j \leqslant r)$ et $y=y^{\nu_{j, i}}\left(1 \leqslant j \leqslant r, 1 \leqslant i \leqslant \alpha_{j}\right)$, fournit, après élévation à la puissance $\kappa_{j, i}$ et et formation du produit,

$$
\sum_{n \geqslant 1} \frac{f_{y}(n)}{n^{s}}=(\log y)^{\kappa} s J_{f}(s) \widehat{\varrho}((s-1) \log y ; \boldsymbol{\kappa}, \boldsymbol{\nu})\left\{1+O\left(\frac{1}{L_{\beta}(y)}\right)\right\},
$$

où $J_{f}(s):=(s-1)^{\kappa} \mathcal{F}(s) / s$.

\section{Applications}

\subsection{Entiers représentables comme somme de deux carrés}

Soit $b(n)$ la fonction indicatrice des entiers représentables comme somme de deux carrés. Dans [20], Tenenbaum \& Wu ont obtenu, pour tout $\beta \in] 0, \frac{3}{5}[$, la formule asymptotique

$$
\Psi_{b}(x, y)=\frac{B x \varrho_{1 / 2}(u)}{\sqrt{\log y}}\left\{1+O\left(\frac{1}{\sqrt{\log y}}+\frac{\log (u+1)}{\log y}\right)\right\} \quad\left((x, y) \in H_{\beta}\right),
$$

où l'on a posé

$$
B:=\sqrt{\frac{\pi}{2}} \prod_{p \equiv 3(\bmod 4)}\left(1-1 / p^{2}\right)^{-1 / 2} .
$$

D'après la formule $(2 \cdot 21)$ de [20], la série de Dirichlet associée à la fonction $b$ vaut

$$
\mathcal{B}(s)=\zeta(s)^{1 / 2} L\left(s, \chi_{4}\right)^{1 / 2}\left(1-2^{-s}\right)^{-1 / 2} \prod_{p \equiv 3(\bmod 4)}\left(1-p^{-2 s}\right)^{-1 / 2},
$$

où $\chi_{4}$ désigne l'unique caractère de Dirichlet non principal de module 4 . Il est facile de voir que, en opérant le choix standard $(1 \cdot 14), b \in \mathcal{H}_{+}\left(\frac{1}{2} ; \beta, c, \delta\right)$ pour tous $\beta>0, \delta>0, \beta+\delta<3 / 5$. L'énoncé suivant résulte donc immédiatement des Théorèmes 1.1 et 1.2 . Nous notons $\left\{a_{j}(b)\right\}_{j=0}^{\infty}$ la suite des coefficients de Taylor à l'origine de $s^{1 / 2} \mathcal{B}(s+1) /(s+1)$. En particulier, $a_{0}(b)=B$. 
Théorème 2.1. Soient $\beta \in] 0,3 / 5[$ et $J \in \mathbb{N}$. Nous avons

$$
\Psi_{b}(x, y)=\frac{x}{\sqrt{\log y}}\left\{\sum_{0 \leqslant j \leqslant J} \frac{a_{j}(b) \varrho_{1 / 2}^{(j)}(u)}{(\log y)^{j}}+O\left(\varrho_{1 / 2}(u)\left\{\frac{\log (u+1)}{\log y}\right\}^{J+1}\right)\right\}
$$

uniformément sous les conditions $\left(G_{\beta}\right)$ et $\mathcal{G}_{J}(1 / 2)$.

Compte tenu de la remarque suivant l'énoncé du Lemme 3.1 infra, nous déduisons de $(2 \cdot 2)$ que le terme d'erreur de $(2 \cdot 1)$ est optimal.

\subsection{Répartition des valeurs de la fonction d'Euler}

Désignons par $n \mapsto \varphi(n)$ la fonction indicatrice d'Euler. Smati \& Wu [12] d'une part, et Naïmi [8] d'autre part, ont obtenu, indépendamment et par des méthodes différentes, l'estimation

$$
\sum_{\substack{\varphi(n) \leqslant x \\ P(n) \leqslant y}} 1=\operatorname{Ex} \varrho(u)\left\{1+O\left(\frac{\log (u+1)}{\log y}\right)\right\} \quad\left((x, y) \in H_{\beta}\right),
$$

pour tout $\beta \in] 0, \frac{3}{5}$, où l'on a posé $E:=\zeta(2) \zeta(3) / \zeta(6)$.

Soit

$$
w(n):=\sum_{\substack{m \geqslant 1 \\ \varphi(m)=n}} 1 \quad(n \geqslant 1) .
$$

La série de Dirichlet associée à la fonction arithmétique $w$ vaut

$$
\sum_{n \geqslant 1} \frac{1}{\varphi(n)^{s}}=\zeta(s) G(s), \quad \text { où } \quad G(s):=\prod_{p}\left(1+\frac{1}{(p-1)^{s}}-\frac{1}{p^{s}}\right) .
$$

Le théorème 2 de [1] et le lemme 2.3 de [12], impliquent immédiatement qu'en choisissant, dans $(1 \cdot 10)$,

$$
g_{y}(n):=\sum_{\substack{P(d m) \leqslant y \\ \varphi(d) m=n}} \mu(m),
$$

on a $w \in \mathcal{H}_{+}(1 ; \beta, c, \delta)$ pour tous $\beta>0, \delta>0, \beta+\delta<3 / 5$. Nous obtenons donc le résultat suivant où $\left\{a_{j}(w)\right\}_{j=0}^{\infty}$ désigne la suite des coefficients de Taylor à l'origine de $s \zeta(s+1) G(s+1) /(s+1)$, de sorte que $a_{0}(w)=E$.

Théorème 2.2. Soient $\beta \in] 0,3 / 5[$ et $J \in \mathbb{N}$. Nous avons

$$
\sum_{\substack{\varphi(n) \leqslant x \\ P(n) \leqslant y}} 1=x \sum_{0 \leqslant j \leqslant J} \frac{a_{j}(w) \varrho^{(j)}(u)}{(\log y)^{j}}+O\left(x \varrho(u)\left\{\frac{\log (u+1)}{\log y}\right\}^{J+1}\right)
$$

uniformément sous les conditions $\left(G_{\beta}\right)$ et $\mathcal{G}_{J}(1)$.

Comme précédemment, nous retrouvons ainsi l'optimalité du terme d'erreur de $(2 \cdot 3)$. 


\subsection{Nombre de solutions de congruences polynomiales modulo les entiers friables}

Soit $F$ un polynôme à coefficients entiers, de degré $g$, prenant des valeurs positives sur les entiers positifs. Écrivons

$$
F(X)=\prod_{1 \leqslant j \leqslant r} F_{j}(X)^{\alpha_{j}}
$$

sa décomposition en produit de facteurs irréductibles, désignons par

$$
F^{*}(X)=\prod_{1 \leqslant j \leqslant r} F_{j}(X) .
$$

son noyau sans facteur carré et, pour chaque entier $n \geqslant 1$, notons $\varrho_{F}(n)$ le nombre des racines de $F$ dans $\mathbb{Z} / n \mathbb{Z}$.

Pour $1 \leqslant j \leqslant r$, donnons-nous un zéro $\vartheta_{j}$ de $F_{j}$ et posons $\mathbb{K}_{j}:=\mathbb{Q}\left(\vartheta_{j}\right)$. Un cas particulier de la formule $(3 \cdot 35)$ de [15] s'écrit

$$
\sum_{n \geqslant 1} \frac{\varrho_{F}(n)}{n^{s}}=\prod_{1 \leqslant j \leqslant r} \prod_{1 \leqslant \nu \leqslant \alpha_{j}} \zeta_{\mathbb{K}_{j}}(1+\nu(s-1)) G_{F}(s) \quad(\sigma>1),
$$

où $G_{F}(s)$ est une série de Dirichlet absolument convergente dans le demi-plan $\sigma>1-\sigma_{0}$ avec $\sigma_{0}:=1 /\left(2 \max _{j} \alpha_{j}\right)$. Cela implique immédiatement que, pour tout $\delta<\sigma_{0}$, la fonction $G_{F}(s)$ satisfait la majoration (1.7) dans le demi-plan $\sigma \geqslant 1-\delta$ et la formule $(1 \cdot 10)$ avec un terme d'erreur $\ll 1 / y^{\delta}$. Dans le cas particulier où tous les $\alpha_{j}$ sont égaux à 1 , nous obtenons

$$
\sum_{n \geqslant 1} \frac{\varrho_{F^{*}}(n)}{n^{s}}=\prod_{1 \leqslant j \leqslant r} \zeta_{\mathbb{K}_{j}}(s) G_{F^{*}}(s) \quad(\sigma>1) .
$$

Ainsi, pour tout $\beta \in] 0,3 / 5$ [ et pour des constantes positives convenables $c$ et $\delta$, nous avons $\varrho_{F} \in \widetilde{\mathcal{H}}_{+}^{*}(r ; \beta, c, \delta)$ et $\varrho_{F^{*}} \in \mathcal{H}_{+}^{*}(r ; \beta, c, \delta)$. Une application directe des Théorèmes 1.1 et 1.2 fournit immédiatement le résultat suivant où $\left\{a_{j}\left(\varrho_{F^{*}}\right)\right\}_{j=0}^{\infty}$ désigne la suite des coefficients de Taylor à l'origine de

$$
\frac{s^{r}}{s+1} \sum_{n \geqslant 1} \frac{\varrho_{F^{*}}(n)}{n^{s+1}} .
$$

Théorème 2.3. Soient $\beta \in] 0,3 / 5[$ et $J \in \mathbb{N}$. Nous avons

$$
\Psi_{\varrho_{F^{*}}}(x, y)=x(\log y)^{r-1}\left\{\sum_{0 \leqslant j \leqslant J} \frac{a_{j}\left(\varrho_{F^{*}}\right) \varrho_{r}^{(j)}(u)}{(\log y)^{j}}+O\left(\varrho_{r}(u)\left\{\frac{\log (u+1)}{\log y}\right\}^{J+1}\right)\right\}
$$

uniformément sous les conditions $\left(H_{\beta}\right)$ et $\mathcal{G}_{J}(r)$.

Le cas général (i.e. $F \neq F^{*}$ ) relève de l'extension décrite au paragraphe 1.4. Nous obtenons alors la validité de $(1 \cdot 27)$ pour $f=\varrho_{F}$ sous les conditions $\left(H_{\beta}\right)$ et $\mathcal{G}_{J}(\kappa)$. 


\subsection{Solubilité de congruences polynomiales modulo les entiers friables}

Soit $F$ un polynôme à coefficients entiers. Définissons le noyau $F^{*}$ comme au paragraphe précédent. Notons $G$ le groupe de Galois du corps de décomposition de $F$. Ce groupe agit par permutation sur les racines de $F$; en particulier, pour chaque élément $\gamma$ de $G$, on peut définir l'entier $n_{\gamma}$ comme le nombre de points fixes de cette action.

Soit $\delta_{F}$ la fonction arithmétique indicatrice de l'ensemble des entiers $n$ tels que $F$ possède au moins une racine dans $\mathbb{Z} / n \mathbb{Z}$. Nous établissons au paragraphe 5 l'identité

$$
\sum_{n \geqslant 1} \frac{\delta_{F}(n)}{n^{s}}=\zeta(s)^{\kappa_{F}} H(s) \quad(\sigma>1)
$$

où $H(s)$ est prolongeable en une fonction holomorphe et sans zéro dans la région $(1 \cdot 6)$, vérifiant les hypothèses $(1 \cdot 7)$ et $(1 \cdot 10)$, et où la constante $\kappa_{F}$ est définie par

$$
\kappa_{F}:=\frac{1}{|G|}\left|\left\{\gamma \in G: n_{\gamma} \neq 0\right\}\right|
$$

Par exemple, lorsque $F=\Phi_{n}$ est le $n$-ième polynôme cyclotomique, on a $\kappa_{F}=1 / \varphi(n)$; lorsque $F(X)=\left(X^{2}-2\right)\left(X^{2}-3\right)\left(X^{2}-6\right)$, on a $\kappa_{F}=1$; et lorsque $F(X)=X^{n}-X-1$, de groupe de Galois le groupe symétrique $S_{n}$ (voir [9]), on trouve

$$
\kappa_{F}=\sum_{1 \leqslant j \leqslant n} \frac{(-1)^{j-1}}{j !} .
$$

Notant $\left\{a_{j}\left(\delta_{F}\right)\right\}_{j=0}^{\infty}$ la suite des coefficients de Taylor à l'origine de

$$
\frac{s^{\kappa_{F}}}{s+1} \sum_{n \geqslant 1} \frac{\delta_{F}(n)}{n^{s+1}}
$$

nous obtenons donc le théorème suivant.

Théorème 2.4. Soient $\beta \in] 0,3 / 5[$ et $J \in \mathbb{N}$. Nous avons

$$
\Psi_{\delta_{F}}(x, y)=x(\log y)^{\kappa_{F}-1}\left\{\sum_{0 \leqslant j \leqslant J} \frac{a_{j}\left(\delta_{F}\right) \varrho_{\kappa_{F}}^{(j)}(u)}{(\log y)^{j}}+O\left(\varrho_{\kappa_{F}}(u)\left\{\frac{\log (u+1)}{\log y}\right\}^{J+1}\right)\right\}
$$

uniformément sous les conditions $\left(G_{\beta}\right)$ et $\mathcal{G}_{J}\left(\kappa_{F}\right)$.

Au paragraphe 5 , nous considérons en fait toute fonction de la forme $\mathcal{J}\left(\varrho_{F^{*}}(n)\right)$, où $\mathcal{J}$ est une fonction arithmétique totalement multiplicative à valeurs réelles 
positives ou nulles. Cela permet d'obtenir un résultat de même type, où $\kappa_{F}$ doit être remplacé par

$$
\kappa_{F, \mathfrak{J}}=\frac{1}{|G|} \sum_{\gamma \in G} \mathcal{J}\left(n_{\gamma}\right) .
$$

Pour $\mathcal{J}(m)=m^{t}(t>0)$, on obtient ainsi une évaluation de la valeur moyenne de chaque moment réel positif de $\varrho_{F^{*}}(n)$ sur les entiers friables.

\section{Développement asymptotique du terme principal}

\subsection{Lemmes}

Notre premier objectif consiste à obtenir une formule asymptotique pour $\Lambda_{f}(x, y)$ lorsque $y \leqslant x \leqslant y^{2}$. Nous définissons les termes d'erreur $\Delta_{f}(t)$ et $\Delta_{f}^{*}(t)$ par les relations

$$
\begin{aligned}
\frac{M\left(\mathrm{e}^{t} ; f\right)}{\mathrm{e}^{t}} & =\frac{a_{0}(f) t^{\kappa-1}}{\Gamma(\kappa)}+\Delta_{f}(t), \\
\int_{0}^{t} \frac{M\left(\mathrm{e}^{v} ; f\right)}{\mathrm{e}^{v}} \mathrm{~d} v & =\sum_{n \leqslant \mathrm{e}^{t}} \frac{f(n)}{n}-\frac{1}{\mathrm{e}^{t}} \sum_{n \leqslant \mathrm{e}^{t}} f(n)=\frac{a_{0}(f) t^{\kappa}}{\Gamma(\kappa+1)}+\Delta_{f}^{*}(t) .
\end{aligned}
$$

Sous l'hypothèse $f \in \mathcal{H}\left(\kappa, \kappa_{0} ; \beta, c, \delta\right)$, la méthode de Selberg-Delange fournit

$$
\Delta_{f}(t) \ll \frac{t^{\kappa-1}}{1+t} \quad(t>0), \quad \text { et } \quad \Delta_{f}^{*}(t) \ll \frac{t^{\kappa}+\mathbf{1}_{[1, \infty[}(\kappa) t}{1+t} \quad(t \geqslant 0) .
$$

Lemme 3.1. Soient $\beta, c, \delta, \kappa, \kappa_{0}$, des nombres réels positifs tels que $\beta+\delta<3 / 5$, et $f \in \mathcal{H}\left(\kappa, \kappa_{0} ; \beta, c, \delta\right)$. On a uniformément pour $1 \leqslant u \leqslant 2, y \geqslant 2$,

$$
\Lambda_{f}(x, y)=a_{0}(f) x(\log y)^{\kappa-1} \varrho_{\kappa}(u)\left\{1+\frac{\kappa \Delta_{f}^{*}(\log (x / y))}{(\log y)^{\kappa}}+O\left(\frac{1}{\log y}\right)\right\} .
$$

En particulier, sous les mêmes hypothèses,

$$
\Lambda_{f}(x, y)=a_{0}(f) x(\log y)^{\kappa-1} \varrho_{\kappa}(u)\left\{1+O\left(\frac{1}{(\log y)^{\min (1, \kappa)}}\right)\right\} .
$$

Remarque. Pour $\log 2 \leqslant t<\log 3, f(1)=1, f(2)=\kappa$, on a

$$
\Delta_{f}^{*}(t)=1+\frac{1}{2} \kappa-(1+\kappa) \mathrm{e}^{-t}-a_{0}(f) t^{\kappa} / \Gamma(\kappa+1) .
$$

Cette fonction possédant au plus deux zéros sur $[\log 2, \log 3[$, il s'ensuit que le terme d'erreur de (3.5) est optimal en toute généralité. 
Démonstration. Par sommation d'Abel, nous déduisons de $(3 \cdot 1)$ et $(3 \cdot 2)$ que l'on a, pour $u \geqslant 1$,

$$
\begin{aligned}
\frac{\Lambda_{f}(x, y)}{x} & =\frac{M\left(y^{u} ; f\right)}{y^{u}}+\frac{1}{\log y} \int_{0-}^{u} z_{\kappa}^{\prime}(u-v) \mathrm{d}\left(\int_{0}^{v} \frac{M\left(y^{w} ; f\right)}{y^{w}} \mathrm{~d}(w \log y)\right) \\
& =a_{0}(f)(\log y)^{\kappa-1} \varrho_{\kappa}(u)+\Delta_{f}(u \log y)+\frac{R}{\log y},
\end{aligned}
$$

où nous avons fait appel à l'identité $(1 \cdot 20)$ sous la forme

$$
\varrho_{\kappa}(u)=\frac{1}{\Gamma(\kappa)}\left(u^{\kappa-1}+\int_{0}^{u} z_{\kappa}^{\prime}(u-v) v^{\kappa-1} \mathrm{~d} v\right) \quad(u>0)
$$

et posé

$$
R:=\int_{0}^{u} z_{\kappa}^{\prime}(u-v) \mathrm{d} \Delta_{f}^{*}(v \log y)
$$

Évaluons $R$ par une nouvelle sommation d'Abel en tenant compte de la discontinuité de première espèce de $z_{\kappa}^{\prime}(v)$ en $v=1$. Grâce à la seconde majoration $(3 \cdot 3)$, nous obtenons, toujours pour $1 \leqslant u \leqslant 2$,

$$
\begin{aligned}
R & =\kappa \Delta_{f}^{*}((u-1) \log y)+\int_{0}^{u} z_{\kappa}^{\prime \prime}(u-v) \Delta_{f}^{*}(v \log y) \mathrm{d} v \\
& =\kappa \Delta_{f}^{*}((u-1) \log y)+O\left((\log y)^{\kappa-1} \int_{0}^{u}\left|z_{\kappa}^{\prime \prime}(u-v)\right| v^{\kappa-1} \mathrm{~d} v\right) \\
& =\kappa \Delta_{f}^{*}((u-1) \log y)+O\left((\log y)^{\kappa-1}\right) .
\end{aligned}
$$

Reportons dans $(3 \cdot 6)$ en incorporant la première majoration $(3 \cdot 3)$. Nous obtenons bien le résultat requis $(3 \cdot 4)$.

Cela achève la démonstration.

Posons, pour $x \geqslant y \geqslant 2, u=(\log x) / \log y$,

$$
\lambda_{y}(u):=\frac{\Lambda_{f}(x, y)}{x}=\int_{\mathbb{R}} z_{\kappa}(u-v) \mathrm{d}\left(\frac{M\left(y^{v} ; f\right)}{y^{v}}\right)
$$

et rappelons les définitions des quantités $m$ et $\vartheta$ en $(1 \cdot 22)$.

Lemme 3.2. Soient $\beta, c, \delta, \kappa$, des nombres réels positifs tels que $\beta+\delta<3 / 5$, et $f$ une fonction arithmétique dont la série de Dirichlet $\mathcal{F}(s)$ appartient à la classe $\mathcal{E}_{\kappa}(\beta, c, \delta)$. Alors, pour $u \geqslant 1$ et $y \geqslant 2$, on a

$$
\lambda_{y}(u)=(\log y)^{\kappa-m-1} \int_{0-}^{u} \varrho_{\kappa}^{(m)}(u-v) \mathrm{d} \nu_{\kappa}(v \log y)
$$

où l'on a posé

$$
\nu_{\kappa}(v):=\frac{1}{\Gamma(1-\vartheta)} \int_{0}^{v^{+}} \frac{M\left(\mathrm{e}^{w} ; f\right)}{\mathrm{e}^{w}(v-w)^{\vartheta}} \mathrm{d} w \quad(v \in \mathbb{R})
$$

et où, lorsque $\kappa=m$, la dérivée $\varrho_{\kappa}^{(m)}$ est comprise au sens des distributions. 
Démonstration. Notant, dans un demi-plan de convergence adéquat,

$$
\widehat{g}(s):=\int_{0}^{\infty} g(t) \mathrm{e}^{-t s} \mathrm{~d} t
$$

la transformée de Laplace d'une fonction $g$, nous avons,

$$
\left\{\begin{array}{l}
\int_{0-}^{\infty} \mathrm{e}^{-s v} \mathrm{~d}\left(M\left(\mathrm{e}^{v} ; f\right) / \mathrm{e}^{v}\right)=s \mathcal{F}(s+1) /(s+1), \\
\widehat{z_{\kappa}}(s)=s^{\kappa-1} \widehat{\varrho_{\kappa}}(s), \\
s^{m} \widehat{\varrho_{\kappa}}(s)=\widehat{\varrho_{\kappa}^{(m)}}(s) .
\end{array}\right.
$$

Il s'ensuit que, notant $s_{y}:=s / \log y$,

$$
\begin{aligned}
\widehat{\lambda_{y}}(s) & =\widehat{z_{\kappa}}(s) \frac{s_{y}}{s_{y}+1} \mathcal{F}\left(s_{y}+1\right)=(\log y)^{\kappa-m-1} s^{m} \widehat{\varrho_{\kappa}}(s) \frac{s_{y}^{\vartheta} \mathcal{F}\left(s_{y}+1\right)}{s_{y}+1} \\
& =(\log y)^{\kappa-m-1} \widehat{\varrho_{\kappa}^{(m)}}(s) \frac{s_{y}^{\vartheta} \mathcal{F}\left(s_{y}+1\right)}{s_{y}+1} .
\end{aligned}
$$

D'autre part, on établit aisément grâce au théorème des résidus que

(3.11) $\frac{1}{2 \pi i} \int_{1-i \infty}^{1+i \infty} \frac{\mathrm{e}^{v s} s^{\vartheta-1}}{s+1} \mathrm{~d} s=h_{\vartheta}(v):=\frac{1}{\Gamma(1-\vartheta)} \int_{0}^{v^{+}} \mathrm{e}^{t-v} t^{-\vartheta} \mathrm{d} t \quad(v \in \mathbb{R})$.

La fonction $h_{\vartheta}$ est continue sur $\mathbb{R}$, dérivable sur $\mathbb{R}^{*}$ et à variation bornée sur tout intervalle borné. Par sommation et inversion de Laplace, la relation (3.11) implique alors

$$
\frac{s^{\vartheta} \mathcal{F}(s+1)}{s+1}=\int_{\mathbb{R}} \mathrm{e}^{-s v} \mathrm{~d} \nu_{\kappa}(v)
$$

avec

$$
\begin{aligned}
\nu_{\kappa}(v) & :=\sum_{n \geqslant 1} \frac{f(n)}{n} h_{\vartheta}(v-\log n) \\
& =\frac{1}{\Gamma(1-\vartheta)} \sum_{n \leqslant \mathrm{e}^{v}} f(n) \int_{0}^{v-\log n} \frac{\mathrm{e}^{t-v}}{t^{\vartheta}} \mathrm{d} t \\
& =\frac{1}{\Gamma(1-\vartheta)} \int_{0}^{v^{+}} \frac{M\left(\mathrm{e}^{v-t} ; f\right)}{t^{\vartheta} \mathrm{e}^{v-t}} \mathrm{~d} t .
\end{aligned}
$$

Le théorème de convolution implique donc bien (3.8). 
Lemme 3.3. Soit $\vartheta \in[0,1[$. On a uniformément pour $T \geqslant 1, w \in \mathbb{R}, \sigma>0$

$$
\frac{1}{2 \pi i} \int_{\sigma-i T}^{\sigma+i T} \frac{\mathrm{e}^{w s} s^{\vartheta-1}}{s+1} \mathrm{~d} s=h_{\vartheta}(w)+O\left(\frac{\mathrm{e}^{\sigma w}}{T^{1-\vartheta}(1+T|w|)}\right) .
$$

Démonstration. Lorsque $|w| T>1$, nous pouvons écrire

$$
\begin{aligned}
\int_{\sigma+i T}^{\sigma+i \infty} \mathrm{e}^{w s} \frac{s^{\vartheta-1}}{s+1} \mathrm{~d} s & =\left[\frac{\mathrm{e}^{w s}}{w} \frac{s^{\vartheta-1}}{s+1}\right]_{\sigma+i T}^{\sigma+i \infty}-\int_{\sigma+i T}^{\sigma+i \infty} \frac{\mathrm{e}^{w s}}{w} \frac{\mathrm{d}}{\mathrm{d} s}\left(\frac{s^{\vartheta-1}}{s+1}\right) \mathrm{d} s \\
& \ll \frac{\mathrm{e}^{\sigma w}}{T^{2-\vartheta} w} .
\end{aligned}
$$

Lorsque $|w| T \leqslant 1$, nous observons que

$$
\int_{\sigma+i T}^{\sigma+i \infty} \frac{\mathrm{e}^{w s} s^{\vartheta-1}}{s+1} \mathrm{~d} s \ll \mathrm{e}^{\sigma w} \int_{\sigma+i T}^{\sigma+i \infty}\left|s^{\vartheta-2}\right||\mathrm{d} s| \ll \mathrm{e}^{\sigma w} T^{\vartheta-1} .
$$

Le lemme suivant fournit une mesure effective du module de continuité de la fonction $\nu_{\kappa}$. Il fait explicitement intervenir une condition sur la distance à l'ensemble des entiers. Nous posons classiquement $\|x\|:=\min _{n \in \mathbb{Z}}|x-n|$.

Lemme 3.4. Soient $\beta, c, \delta, \kappa$, des nombres réels positifs tels que $\beta+\delta<3 / 5$, et $f \in \mathcal{H}_{+}(\kappa ; \beta, c, \delta)$. Nous avons, uniformément pour $v \geqslant 3,\left\|\mathrm{e}^{v}\right\| \gg 1$,

$$
\nu_{\kappa}(v)-\nu_{\kappa}(v-h) \ll h^{1-\vartheta} L_{\beta}\left(\mathrm{e}^{v} / h\right)^{-\vartheta}+v^{\kappa_{0}} h \quad\left(v \geqslant 3,0 \leqslant h \leqslant \frac{1}{2}\right) .
$$

Démonstration. Commençons par observer que l'hypothèse concernant la série majorante de $\mathcal{F}(s)$ fournit, par la méthode de Selberg-Delange (voir [16], chap. II.5 et II.6), la majoration

$$
\sum_{x<n \leqslant x+z}|f(n)| \ll z(\log x)^{\kappa_{0}-1}+x / L_{\beta}(x)^{10} \quad(1 \leqslant z \leqslant x) .
$$

Notons, à fins de référence ultérieure, que cela implique

$$
|f(n)| \ll n / L_{\beta}(n)^{10} \quad(n \geqslant 1) .
$$

Considérons en premier lieu le cas général où $\left\|\mathrm{e}^{v}\right\|$ n'est pas minoré. Soit $N$ un entier naturel tel que $\left\|\mathrm{e}^{v}\right\|=\left|\mathrm{e}^{v}-N\right|$. Pour $v \geqslant 3, n \in \mathbb{N}^{*}$, spécialisons, dans (3.13), $\sigma=1 / v, w=v-\log n$, multiplions par $f(n) / n$ et sommons sur $n \in \mathbb{N}^{*}$. Nous obtenons

$$
\nu_{\kappa}(v)=\frac{1}{2 \pi i} \int_{1 / v-i T}^{1 / v+i T} \frac{s^{\vartheta} \mathcal{F}(s+1) \mathrm{e}^{v s}}{s(s+1)} \mathrm{d} s+O\left(\frac{1}{T^{1-\vartheta}} \sum_{n \geqslant 1} \frac{|f(n)|}{n^{1+1 / v}(1+T|v-\log n|)}\right) .
$$


La contribution à la dernière somme en $n$ des entiers $n$ n'appartenant pas à $[N / 2,3 N / 2]$ est $\ll v^{\kappa_{0}} / T$. Celle du terme d'indice $N$ est, en vertu de $(3 \cdot 16)$,

$$
\ll \frac{\mathrm{e}^{v}}{\left(\mathrm{e}^{v}+T\left\|\mathrm{e}^{v}\right\|\right) L_{\beta}\left(\mathrm{e}^{v}\right)^{10}} .
$$

Notons $V$ la contribution complémentaire, de sorte que

$$
V \ll \sum_{\substack{N / 2 \leqslant n \leqslant 3 N / 2 \\ n \neq N}} \frac{|f(n)|}{n+T|N-n|} .
$$

Lorsque $T>N$, cela implique

$$
\begin{aligned}
V & \ll \frac{1}{T} \sum_{0 \leqslant m \leqslant L_{\beta}(N)^{10}} \frac{1}{m+1} \sum_{\left|n-N-m N / L_{\beta}(N)^{10}\right|<N / L_{\beta}(N)^{10}}|f(n)| \\
& \ll \frac{N(\log N)^{\beta+\kappa_{0}}}{T L_{\beta}(N)^{10}} \ll \frac{1}{L_{\beta}\left(\mathrm{e}^{v} T\right)^{4}} .
\end{aligned}
$$

Lorsque $T \leqslant N$, nous pouvons écrire

$$
\begin{aligned}
V & \ll \sum_{0 \leqslant m \leqslant T / 2} \frac{1}{(m+1) N} \sum_{m N / T<|N-n| \leqslant(m+1) N / T}|f(n)| \\
& \ll \sum_{0 \leqslant m \leqslant T / 2} \frac{1}{(m+1) N}\left\{\frac{N(\log N)^{\kappa_{0}-1}}{T}+\frac{N}{L_{\beta}(N)^{10}}\right\} \\
& \ll \frac{v^{\kappa_{0}}}{T}+\frac{1}{L_{\beta}\left(\mathrm{e}^{v} T\right)^{4}} .
\end{aligned}
$$

Nous obtenons ainsi

$$
\begin{aligned}
\nu_{\kappa}(v)= & \frac{1}{2 \pi i} \int_{(1 / v)-i T}^{(1 / v)+i T} \frac{s^{\vartheta} \mathcal{F}(s+1) \mathrm{e}^{v s}}{s(s+1)} \mathrm{d} s \\
& +O\left(\frac{v^{\kappa_{0}}}{T^{2-\vartheta}}+\frac{1}{T^{1-\vartheta} L_{\beta}\left(\mathrm{e}^{v} T\right)^{4}}+\frac{\mathrm{e}^{v}}{T^{1-\vartheta}\left(\mathrm{e}^{v}+T\left\|\mathrm{e}^{v}\right\| L_{\beta}\left(\mathrm{e}^{v}\right)^{10}\right.}\right) .
\end{aligned}
$$

La même formule vaut pour $\nu_{\kappa}(v-h)$, en remplaçant seulement $\mathrm{e}^{v s}$ par $\mathrm{e}^{(v-h) s}$ dans l'intégrande et $\left\|\mathrm{e}^{v}\right\|$ par $\left\|\mathrm{e}^{v-h}\right\|$ dans le membre de droite. D'où, sous les conditions de l'énoncé,

$$
\begin{aligned}
\nu_{\kappa}(v)-\nu_{\kappa}(v-h) \ll & \int_{(1 / v)-i T}^{(1 / v)+i T} \frac{|\mathcal{F}(s+1)||s|^{\vartheta}}{|s+1|}|\mathrm{d} s|+\frac{v^{\kappa_{0}}}{T^{2-\vartheta}} \\
& +\frac{1}{T^{1-\vartheta} L_{\beta}\left(\mathrm{e}^{v} T\right)^{4}}+\frac{\mathrm{e}^{v}}{T^{1-\vartheta}\left(\mathrm{e}^{v}+T\left\|\mathrm{e}^{v-h}\right\|\right) L_{\beta}\left(\mathrm{e}^{v}\right)^{10}} \\
\ll & h T^{\vartheta}(\log T)^{\kappa}+h v^{\kappa_{0}-\vartheta}+\frac{v^{\kappa_{0}}}{T^{2-\vartheta}}+\frac{1}{T^{1-\vartheta} L_{\beta}\left(\mathrm{e}^{v} T\right)^{4}} \\
& +\frac{\mathrm{e}^{v}}{T^{1-\vartheta}\left(\mathrm{e}^{v}+T\left\|\mathrm{e}^{v-h}\right\|\right) L_{\beta}\left(\mathrm{e}^{v}\right)^{10}} .
\end{aligned}
$$


Il existe une constante $c_{3}$ telle que $\left\|\mathrm{e}^{v-h}\right\| \gg 1$ si $h \leqslant c_{3} \mathrm{e}^{-v}$. Dans ce cas, le dernier terme de cette majoration est

$$
\ll \frac{\mathrm{e}^{v}}{T^{2-\vartheta} L_{\beta}\left(\mathrm{e}^{v}\right)^{10}} \ll \frac{1 / h}{T^{2-\vartheta} L_{\beta}\left(\mathrm{e}^{v} / h\right)^{5}} .
$$

Dans le cas contraire, il est

$$
\frac{\mathrm{e}^{v}}{T^{1-\vartheta}\left(\mathrm{e}^{v}+T\left\|\mathrm{e}^{v-h}\right\|\right) L_{\beta}\left(\mathrm{e}^{v}\right)^{10}} \leqslant \frac{1}{T^{1-\vartheta} L_{\beta}\left(\mathrm{e}^{v}\right)^{10}} \ll \frac{1}{T^{1-\vartheta} L_{\beta}\left(\mathrm{e}^{v} / h\right)^{5}} .
$$

Nous avons donc en toute circonstance

$$
\begin{aligned}
\nu_{\kappa}(v)-\nu_{\kappa}(v-h) \ll & h T^{\vartheta}(\log T)^{\kappa}+v^{\kappa_{0}}\left\{h+\frac{1}{T^{2-\vartheta}}\right\} \\
& +\frac{1}{T^{1-\vartheta} L_{\beta}\left(\mathrm{e}^{v} T\right)^{4}}+\frac{1+1 /(h T)}{T^{1-\vartheta} L_{\beta}\left(\mathrm{e}^{v} / h\right)^{4}} .
\end{aligned}
$$

La majoration annoncée résulte de cette estimation en choisissant par exemple $T:=(1 / h) L_{\beta}\left(\mathrm{e}^{v} / h\right)^{-2}$.

Notre dernier lemme fournit, par intégration complexe, une évaluation précise de $\nu_{\kappa}(v)$ qui semble difficilement accessible par étude directe de la convolution. Il constitue un élément crucial de la démonstration du Théorème 1.1.

Lemme 3.5. Soient $\beta, c, \delta, \kappa$, des nombres réels positifs tels que $\beta+\delta<3 / 5$, et $f$ une fonction arithmétique dont la série de Dirichlet $\mathcal{F}(s)$ appartient à la classe $\mathcal{E}_{\kappa}(\beta, c, \delta)$. S'il existe $\delta_{1}>\vartheta$ tel que l'hypothèse (1.7) soit satisfaite avec $\delta_{1}$ au lieu de $\delta$, alors on a

$$
\nu_{\kappa}(v)=\sum_{0 \leqslant j \leqslant m} a_{m-j}(f) \frac{v^{j}}{j !}+O\left(\frac{1}{L_{\beta}(v)}\right) \quad(v \geqslant 0) .
$$

Si $\kappa \in \mathbb{N}^{*}$ et $\mathcal{F} \in \mathcal{E}_{\kappa}^{*}(\delta)$, la formule (3.18) est valable avec un terme résiduel $\ll \mathrm{e}^{-c_{0} v}$ où $c_{0}$ est une constante positive convenable.

De plus, s'il existe $\kappa_{0}>0$ tel que $f \in \mathcal{H}\left(\kappa, \kappa_{0} ; \beta, c, \delta\right)$, on peut remplacer la condition $\delta_{1}>\vartheta$ par $\delta_{1}>0$.

Démonstration. Nous pouvons pleinement supposer $v \geqslant 2 / c$. D'après $(3 \cdot 12)$, nous pouvons écrire

$$
\nu_{\kappa}(v)=\frac{1}{2 \pi i} \int_{(1 / v)-i \infty}^{(1 / v)+i \infty} \frac{s^{\vartheta} \mathcal{F}(s+1) \mathrm{e}^{v s}}{s(s+1)} \mathrm{d} s .
$$

Notons $s=\sigma+i \tau$ et introduisons un paramètre réel $T \geqslant 3$ que nous fixerons plus loin. Nous pouvons estimer la contribution du domaine $|\tau|>T$ à l'intégrale 
de (3.19) grâce aux majorations classiques de $\zeta(s)$ et à l'hypothèse $(1 \cdot 7)$ modifiée comme indiqué dans l'énoncé. Elle est

$$
\ll T^{-\left(\delta_{1}-\vartheta\right) / 2} .
$$

Si, de plus, $f \in \mathcal{H}\left(\kappa, \kappa_{0} ; \beta, c, \delta\right)$, nous déduisons de (3·17) que cette contribution est

$$
\ll \frac{v^{\kappa_{0}}}{T^{2-\vartheta}}+\frac{1}{T^{1-\vartheta}} .
$$

Nous estimons la contribution complémentaire en déplaçant le segment d'intégration vers la gauche jusqu'à la courbe $\sigma=-c /\left\{2+\log ^{+}|\tau|\right\}^{(1-\beta-\delta) /(\beta+\delta)}$ et en faisant apparaître un contour de Hankel autour de $s=0$. La contribution des segments horizontaux n'excède pas (3·20) et celle de la partie curviligne est

$$
\ll \mathrm{e}^{-c v /\left\{2(\log T)^{(1-\beta-\delta) /(\beta+\delta)}\right\}} .
$$

En choisissant $T=\mathrm{e}^{v^{\beta+\delta}}$, nous obtenons, pour une constante $c_{4}>0$ convenable,

$$
\nu_{\kappa}(v)-I_{\kappa}(v) \ll L_{\beta+\delta}(v)^{-c_{4}} \ll L_{\beta}(v)^{-1},
$$

avec

$$
I_{\kappa}(v):=\frac{1}{2 \pi i} \int_{\mathcal{H}(c / 2)} \frac{s^{\vartheta} \mathcal{F}(s+1) \mathrm{e}^{v s}}{s(s+1)} \mathrm{d} s
$$

et où $\mathcal{H}(X)$ désigne la partie d'un contour de Hankel tournant autour de $s=0$ dans le sens trigonométrique et située dans le demi-plan $\Re e s>-X$. Pour estimer $I_{\kappa}(v)$, nous récrivons $(1 \cdot 16)$ sous la forme du développement de Laurent

$$
\frac{s^{\vartheta} \mathcal{F}(s+1)}{s+1}=\sum_{j=0}^{\infty} a_{j}(f) s^{j-m},
$$

valable pour $0<|s| \leqslant \frac{1}{2} c$. En utilisant, par exemple, la majoration $\left|a_{j}(f)\right| \ll$ $(2 / c)^{j}$, il suit, pour tout entier $N \geqslant 1$,

$$
I_{\kappa}(v)=\sum_{0 \leqslant j \leqslant N+m} \frac{a_{j}(f)}{v^{j-m}} G_{j}(v)+O\left(E_{N}(v)\right)
$$

avec

$$
G_{j}(v):=\frac{1}{2 \pi i} \int_{\mathcal{H}(c v / 2)} s^{j-m-1} \mathrm{e}^{s} \mathrm{~d} s \quad(0 \leqslant j \leqslant N+m)
$$

et

$$
E_{N}(v):=\frac{(2 / c)^{N}}{v^{N+1}} \int_{\mathcal{H}(c v / 2)}|s|^{N} \mathrm{e}^{\sigma}|\mathrm{d} s| .
$$


D'après le corollaire II.5.1 de [16], on a

$$
G_{j}(v)-\frac{1}{\Gamma(m+1-j)} \ll \frac{47^{|m-j|}|m-j| !}{\mathrm{e}^{v / 2}}
$$

et la même technique fournit

$$
E_{N}(v) \ll \frac{(100 / c)^{N} N !}{v^{N+1} \mathrm{e}^{v / 2}} .
$$

Choisissons alors $N=\left[c^{\prime} v\right]+1$ où $c^{\prime}$ est une constante absolue assez petite. Nous obtenons

$$
I_{\kappa}(v)=\sum_{0 \leqslant j \leqslant m} a_{m-j}(f) \frac{v^{j}}{j !}+O\left(\mathrm{e}^{-v / 3}\right) .
$$

Compte tenu de $(3 \cdot 21)$, cela implique bien l'estimation annoncée $(3 \cdot 18)$.

Lorsque $\kappa \in \mathbb{N}^{*}$ et $\mathcal{F} \in \mathcal{E}_{\kappa}^{*}(\delta)$, nous pouvons déplacer, dans l'intégrale de (3·19), le segment d'intégration $[1 / v-i T, 1 / v+i T]$ jusqu'au segment $[-\delta-i T,-\delta+i T]$ avec à présent $T:=\mathrm{e}^{c_{5} v}$ pour une constante convenable $c_{5}>0$. Cela fournit un terme d'erreur $\ll \mathrm{e}^{-c_{0} v}$ dans $(3 \cdot 21)$ et établit ainsi le renforcement indiqué dans l'énoncé.

\subsection{Preuve du Théorème 1.1}

Considérons dans un premier temps le cas $f \in \mathcal{H}\left(\kappa, \kappa_{0} ; \beta, c, \delta\right)$.

Comme la validité de $(1.24)$ sous la condition $\left(G_{\beta}\right)$ résulte de $(3.5)$ et du cas $J=0$ de $(1 \cdot 25)$, nous pouvons nous borner à établir $(1 \cdot 25)$ sous les hypothèses de l'énoncé.

Compte tenu de l'hypothèse $u \in \mathcal{G}_{J}(\kappa)$ et des propriétés de continuité de $\varrho_{\kappa}$ et de ses dérivées, nous pouvons supposer que $y^{u}$ est demi-entier. Nous supposons également que $J \geqslant m$ : dans le cas contraire, il suffit de considérer les termes principaux d'indices supérieurs à $m$ comme des termes d'erreur.

Pour chaque nombre réel $u>0$, désignons par $\xi(u)$ l'unique solution réelle non nulle de l'équation $\mathrm{e}^{\xi}=1+u \xi$ si $u \neq 1$ et posons $\xi(1)=0$. Soit encore

$$
\xi_{\kappa}(u):=\max (1, \xi(u / \kappa)) \quad(u>0)
$$

Sous la condition $\mathcal{G}_{J}(\kappa)$, l'estimation

$$
\varrho_{\kappa}^{(j)}(u-v) \ll \varrho_{\kappa}(u)\{\log (u+1)\}^{j} \mathrm{e}^{v \xi_{\kappa}(u)} \quad\left(0 \leqslant v \leqslant \frac{1}{2} \varepsilon_{J, y}, 0 \leqslant j \leqslant J+1\right)
$$

résulte aisément des évaluations établies dans [14] pour la fonction $\varrho_{\kappa}$ et ses dérivées, ou des formules plus générales données dans [5]. De plus, si $j \leqslant m$, la majoration $(3 \cdot 22)$ est valable pour $0 \leqslant v \leqslant u-\frac{1}{2}$. 
Posons

$$
R_{\kappa}(v):=\nu_{\kappa}(v)-\sum_{0 \leqslant j \leqslant m} a_{m-j}(f) \frac{v^{j}}{j !} .
$$

Conservant la convention d'interpréter $\varrho_{\kappa}^{(m)}$ au sens des distributions lorsque $\kappa=m$ (et donc $\vartheta=0$, ce qui rend la mesure $\mathrm{d} \nu_{\kappa}(v)$ absolument continue), nous avons, d'après $(3 \cdot 7)$,

$$
\frac{\lambda_{y}(u)}{(\log y)^{\kappa-1}}=\sum_{0 \leqslant j<m} \frac{a_{m-j-1}(f) b_{j}(u)}{j !(\log y)^{m-j-1}}+\int_{0-}^{u} \frac{\varrho_{\kappa}^{(m)}(u-v)}{(\log y)^{m}} \mathrm{~d} R_{\kappa}(v \log y)
$$

avec

$$
b_{j}(u):=\int_{0}^{u} v^{j} \varrho_{\kappa}^{(m)}(u-v) \mathrm{d} v=j ! \varrho_{\kappa}^{(m-j-1)}(u) \quad(0 \leqslant j<m) .
$$

Nous pouvons donc écrire

$$
\lambda_{y}(u)=(\log y)^{\kappa-1}\left\{\sum_{0 \leqslant j<m} \frac{a_{j}(f) \varrho_{\kappa}^{(j)}(u)}{(\log y)^{j}}+J_{1}+J_{2}+J_{3}\right\}
$$

où les quantités $J_{\ell}$ correspondent aux contributions respectives à l'intégrale de $(3 \cdot 23)$ des domaines d'intégration $\left[0-, \frac{1}{2} \varepsilon_{J, y}\left[,\left[\frac{1}{2} \varepsilon_{J, y}, u-\frac{1}{2}\left[\right.\right.\right.\right.$ et $\left[u-\frac{1}{2}, u\right]$, la quantité $\varepsilon_{J, y}$ étant définie par $(1 \cdot 23)$.

Nous évaluons $J_{1}$ en utilisant le fait que, sous les hypothèses indiquées, $\varrho_{\kappa}$ est de classe $e^{J+1} \operatorname{sur}\left[u-\frac{1}{2} \varepsilon_{J, y}, u+0\right]$. Pour $0 \leqslant v \leqslant \frac{1}{2} \varepsilon_{J, y}$, la formule de Taylor-Lagrange s'écrit

$$
\begin{aligned}
\varrho_{\kappa}^{(m)}(u-v)= & \sum_{m \leqslant j \leqslant J} \frac{(-1)^{j-m}}{(j-m) !} \varrho_{\kappa}^{(j)}(u) v^{j-m} \\
& +\frac{(-1)^{J-m+1}}{(J-m) !} \int_{0}^{v}(v-w)^{J-m} \varrho_{\kappa}^{(J+1)}(u-w) \mathrm{d} w
\end{aligned}
$$

Reportons dans l'intégrale $J_{1}$ en notant que la représentation (3.12) et l'estimation (3.18) impliquent, grâce à une intégration par parties, que l'on a pour chaque entier naturel fixé $j \geqslant m$,

$$
\frac{(-1)^{j-m}}{(j-m) !} \int_{0-}^{z} v^{j-m} \mathrm{~d} R_{\kappa}(v \log y)=\frac{a_{j}(f)}{(\log y)^{j-m}}+O\left(\frac{1}{L_{\beta}\left(y^{z}\right)}\right) \quad(z \geqslant 0) .
$$

Nous obtenons

$$
J_{1}=\sum_{m \leqslant j \leqslant J} \frac{a_{j}(f) \varrho_{\kappa}^{(j)}(u)}{(\log y)^{j}}+O\left(\varrho_{\kappa}(u)\left\{\frac{\log (u+1)}{\log y}\right\}^{J+1}\right) .
$$


Nous traitons $J_{2}$ et $J_{3}$ comme des termes d'erreur. En utilisant $(3 \cdot 22)$ avec $j=m$ et la monotonie de $\varrho_{\kappa}^{(m)}$, qui peut être établie comme dans [18] où le cas $m=0$ est traité, nous pouvons écrire, grâce à $(3 \cdot 18)$ appliquée au couple $(\beta+\delta / 2, \delta / 2)$,

$$
\begin{aligned}
J_{2} & \ll \varrho_{\kappa}(u)\left\{\frac{\log (u+1)}{\log y}\right\}^{m} \int_{\varepsilon_{J, y}}^{u-1 / 2} \mathrm{e}^{v \xi_{\kappa}(u)-3(v \log y)^{\beta}} \mathrm{d} v \\
& \ll \frac{\varrho_{\kappa}(u)}{\mathrm{e}^{\left(\varepsilon_{J, y} \log y\right)^{\beta}}} \ll \frac{\varrho_{\kappa}(u)}{(\log y)^{J+1}} .
\end{aligned}
$$

Nous évaluons $J_{3}$ par intégration par parties. Nous pouvons supposer $\vartheta>0$ car $\varrho_{\kappa}^{(m)}(v)$ est identiquement nulle sur $[u-1 / 2, u]$ dans le cas contraire. Posant

$$
N_{y}(v):=R_{\kappa}(v \log y)-R_{\kappa}(u \log y)
$$

il suit

$$
J_{3} \ll\left|N_{y}\left(u-\frac{1}{2}\right)\right|+\int_{u-1 / 2}^{u} \frac{\left|N_{y}(v)\right|}{(u-v)^{2-\vartheta}} \mathrm{d} v .
$$

Scindons l'intégrale à $u-\eta / \log y$ où $\eta$ est un paramètre à choisir dans l'intervalle ]0, $\left.\frac{1}{2}\right]$. Nous obtenons, en appliquant les Lemmes 3.4 et 3.5 au couple $(\beta+\delta / 2, \delta / 2)$ au lieu de $(\beta, \delta)$,

$$
\begin{aligned}
J_{3} & \ll \frac{(\log y)^{1-\vartheta}}{L_{\beta+\delta / 2}\left(y^{u}\right)^{2} \eta^{1-\vartheta}}+(\log y)^{1-\vartheta} \int_{0}^{\eta}\left\{L_{\beta+\delta / 2}\left(y^{u} / t\right)^{-\vartheta}+t^{\vartheta}(u \log y)^{\kappa_{0}}\right\} \frac{\mathrm{d} t}{t} \\
& \ll \frac{(\log y)^{1-\vartheta}}{L_{\beta+\delta / 2}\left(y^{u}\right)^{2} \eta^{1-\vartheta}}+L_{\beta+\delta / 2}\left(y^{u}\right)^{-\vartheta / 2}+\eta^{\vartheta}(u \log y)^{\kappa_{0}+1} .
\end{aligned}
$$

En choisissant $\eta:=1 / L_{\beta+\delta / 2}\left(y^{u}\right)$, nous obtenons que $J_{3}$ n'excède pas l'ordre de grandeur du terme résiduel de $(3 \cdot 24)$. Cela achève la preuve de la relation $(1 \cdot 25)$.

Pour traiter le cas $f \in \mathcal{H}^{*}\left(\kappa, \kappa_{0} ; \delta\right)$, nous reprenons les calculs précédents en choisissant

$$
\varepsilon_{J, y}:=B(J+1)\left(\log _{2} y\right) / \log y
$$

pour une constante $B$ assez grande, en faisant appel à la majoration

$$
R_{\kappa}(v) \ll \mathrm{e}^{-c_{0} v}
$$

issue du Lemme 3.5 et en utilisant le fait que $\varrho_{\kappa}^{(j)}(v)=0$ pour $j>m$ et $0 \leqslant v \leqslant 1$. Nous omettons les détails de la vérification. 


\section{Approximation de $\Psi_{f}(x, y)$}

\subsection{Objectif}

Nous nous proposons ici de prouver le Théorème 1.2. Les détails étant très voisins de ceux de la démonstration du théorème III.5.9 de [16] lorsque $\kappa \in \mathbb{N}^{*}$ et de celle du théorème 2 de $[14]$ lorsque $\kappa \in \mathbb{R}^{*+} \backslash \mathbb{N}^{*}$, nous nous limiterons à des indications relativement succinctes.

\subsection{Lemmes}

La série de Dirichlet $\mathcal{F}(s)$ étant définie par (1.8), nous introduisons la troncature

$$
\mathcal{F}(s ; y):=\sum_{n \geqslant 1} f_{y}(n) / n^{s} \quad(y \geqslant 2)
$$

où $f_{y}$ est définie par $(1 \cdot 13)$, et nous posons

$$
J_{f}(s):=\frac{(s-1)^{\kappa} \mathcal{F}(s)}{s},
$$

qui définit une fonction holomorphe dans le domaine (1.6). De plus, si $\kappa \in \mathbb{N}^{*}, J_{f}$ est holomorphe pour $\sigma \geqslant 1-\delta$.

Lemme 4.1. Soient $\beta, c, \delta, \kappa$, des nombres réels positifs tels que $\beta+\delta<3 / 5$ et $\mathcal{F} \in \mathcal{E}_{\kappa}(\beta, c, \delta)$, vérifiant la condition (1·10). Lorsque $(s, y)$ satisfait (1·11), nous avons

$$
\mathcal{F}(s ; y)=(\log y)^{\kappa} s J_{f}(s) \widehat{\varrho_{\kappa}}((s-1) \log y)\left\{1+O\left(\frac{1}{L_{\beta}(y)}\right)\right\} .
$$

Démonstration. Pour chaque corps de nombres $\mathbb{K}$, la fonction zêta de Dedekind $\zeta_{\mathbb{K}}(s)$ possède une région sans zéro de même nature que celle de la fonction $\zeta(s)$ de Riemann. Dans cette région sans zéro, $\log \zeta_{\mathbb{K}}(s)$ relève essentiellement des mêmes estimations que $\log \zeta(s)$ — voir [7]. Il s'ensuit que la démonstration du lemme III.5.9.1 de [16] est encore valable, mutatis mutandis, pour $\zeta_{\mathbb{K}}(s)$. Nous obtenons ainsi

$$
\zeta_{\mathbb{K}}(s ; y)=\zeta_{\mathbb{K}}(s)(s-1)(\log y) \widehat{\varrho}((s-1) \log y)\left\{1+O\left(\frac{1}{L_{\beta}(y)}\right)\right\},
$$

où $\varrho=\varrho_{1}$ est la fonction de Dickman et $\varrho(s)$ désigne sa transformé de Laplace. Lorsque $s \notin] 0,1]$, appliquons cette relation pour $\mathbb{K}=\mathbb{K}_{j}(1 \leqslant j \leqslant r)$, élevons-la à la puissance $\kappa_{j}$, et formons le produit. Compte tenu de la relation $\{\widehat{\varrho}(s)\}^{\kappa}=\widehat{\varrho_{\kappa}}(s)$ et de l'hypothèse $(1 \cdot 10)$, nous obtenons bien le résultat annoncé. Lorsque l'on a $\left.s \in] 1-c /(\log y)^{1-\beta}, 1\right]$, un prolongement par continuité montre que la formule est encore valable.

Posons

$$
\alpha_{0}=\alpha_{0}(x, y):=1-\xi_{\kappa}(u) / \log y
$$


Lemme 4.2. Soient $\beta, c, \delta, \kappa$, des nombres réels positifs tels que $\beta+\delta<3 / 5$, et $\mathcal{F} \in \mathcal{E}_{\kappa}(\beta, c, \delta)$. On suppose la condition (1.10) satisfaite. Sous la condition $\left(H_{\beta}\right)$, nous avons

$$
x^{\alpha_{0}} \mathcal{F}\left(\alpha_{0} ; y\right) \asymp x \varrho_{\kappa}(u) \sqrt{u}(\log y)^{\kappa} .
$$

Démonstration. C'est une conséquence immédiate de $(4 \cdot 1)$ et de la formule $(3 \cdot 25)$ de [20] i.e.

$$
\varrho_{\kappa}(u) \asymp \frac{\mathrm{e}^{-u \xi_{\kappa}(u)} \widehat{\varrho_{\kappa}}\left(-\xi_{\kappa}(u)\right)}{\sqrt{u}} .
$$

Lemme 4.3. Soient $\beta, c, \delta, \kappa$, des nombres réels positifs tels que $\beta+\delta<3 / 5$, et $f \in \mathcal{H}_{+}(\kappa ; \beta, c, \delta)$. Nous avons, uniformément pour $(x, y) \in\left(H_{\beta}\right), T:=L_{\beta+\delta / 2}(y)$,

$$
M\left(x+x / T ; f_{y}\right)-M\left(x ; f_{y}\right) \ll \frac{x \varrho_{\kappa}(u)}{L_{\beta}(y)} .
$$

Démonstration. Nous pouvons supposer, sans perte de généralité, que $y$ est assez grand. Procédons essentiellement comme au lemme III.5.9.4 de [16], en observant que le Lemme 4.1 et l'estimation

$$
\widehat{\varrho_{\kappa}}(s) \asymp 1 / s^{\kappa} \quad\left(s=-\xi_{\kappa}(u)+i \tau,|\tau|>1+u \xi_{\kappa}(u)\right)
$$

impliquent, sous la condition $\left(H_{\beta}\right)$, avec la notation $(4 \cdot 3)$,

$$
\mathcal{F}\left(\alpha_{0}+i \tau ; y\right) \ll\left\{1+|\tau|^{1-\delta / 2}\right\}(\log y)^{\kappa} \quad\left(\frac{1+u \xi_{\kappa}(u)}{\log y} \leqslant|\tau| \leqslant T\right) .
$$

Supposons d'abord $u>B(\log y)^{\beta}$ où $B$ est une constante assez grande. Nous avons alors, en posant $V:=L_{\beta}(y)^{4}$,

$$
\begin{aligned}
M\left(x+x / T ; f_{y}\right)-M\left(x ; f_{y}\right) & \ll \frac{1}{V} \int_{0}^{V} x^{\alpha_{0}}\left|\mathcal{F}\left(\alpha_{0}+i \tau ; y\right)\right| \mathrm{d} \tau \\
& \ll \frac{x^{\alpha_{0}} \mathcal{F}\left(\alpha_{0} ; y\right)}{\sqrt{V}}+x^{\alpha_{0}} V^{1-\delta / 2}(\log y)^{\kappa} \\
& \ll \frac{x \varrho_{\kappa}(u)(\log y)^{\kappa} \sqrt{u}}{\sqrt{V}}+\frac{x \varrho_{\kappa}(u) V}{\mathrm{e}^{u / 2}} \ll \frac{x \varrho_{\kappa}(u)}{L_{\beta}(y)} .
\end{aligned}
$$

Lorsque $u \leqslant B(\log y)^{\beta}$, nous utilisons (3.15) sous la forme

$$
M\left(x+x / T ; f_{y}\right)-M\left(x ; f_{y}\right) \ll \frac{x(\log x)^{\kappa-1}}{T} .
$$

Comme $u \xi_{\kappa}(u)<\frac{1}{2} \log T$ sous les conditions indiquées, cela fournit bien le résultat annoncé.

Le lemme suivant est essentiel pour la démonstration du Théorème 1.2. Nous rappelons la définition de $v_{f}$ en $(1 \cdot 9)$. 
Lemme 4.4. (i) Soient $\beta, c, \delta, \kappa$, des nombres réels positifs tels que $\beta+\delta<3 / 5$, et $f \in \mathcal{H}\left(\kappa, \kappa_{0} ; \beta, c, \delta\right)$. Pour

$$
|\tau|>3, \quad 1-\frac{c}{(\log |\tau|)^{(1-\beta) / \beta}}<\sigma<1, \quad s=\sigma+i \tau, \quad c \log N \geqslant(\log |\tau|)^{1 / \beta},
$$

on a

$$
\sum_{n \leqslant N} \frac{f(n)}{n^{s}}=\mathcal{F}(s)+O\left(\frac{N^{1-\sigma}(\log N)^{\kappa_{0}+1}}{|\tau|^{\mid \delta / 2}}\right) .
$$

(ii) Soient $\delta \in] 0,1], \kappa \in \mathbb{N}^{*}, \kappa_{0} \in \mathbb{N}^{*}, f \in \mathcal{H}^{*}\left(\kappa, \kappa_{0} ; \delta\right)$. Pour

$$
1-\delta /\left(v_{f}+2\right)<\sigma<1, \quad|\tau|>3, \quad s=\sigma+i \tau, \quad N \geqslant|\tau|^{\left(v_{f}+2\right) / \delta},
$$

on a

$$
\sum_{n \leqslant N} \frac{f(n)}{n^{s}}=\mathcal{F}(s)+O\left(\frac{N^{1-\sigma}(\log N)^{\kappa_{0}}}{|\tau|^{2 \delta /\left(v_{f}+2\right)}}\right) .
$$

Démonstration. Posons $T:=\frac{1}{2}|\tau|$. D'après le théorème II.2.2 de [16], nous pouvons écrire, avec $\alpha=1-\sigma+1 / \log N$,

$$
\sum_{n \leqslant N} \frac{f(n)}{n^{s}}=\frac{1}{2 \pi i} \int_{\alpha-i T}^{\alpha+i T} \mathcal{F}(w+s) \frac{N^{w}}{w} \mathrm{~d} w+R(N, T)
$$

avec

$$
R(N, T) \ll N^{1-\sigma} \sum_{n=1}^{\infty} \frac{|f(n)| n^{-\alpha-\sigma}}{1+T|\log (N / n)|} .
$$

La contribution au membre de droite des entiers $n$ tels que $|\log (N / n)|>1$ est

$$
\ll \frac{N^{1-\sigma}}{T} \sum_{n \geqslant 1} \frac{|f(n)|}{n^{\alpha+\sigma}} \ll \frac{N^{1-\sigma}(\log N)^{\kappa_{0}}}{T} .
$$

Pour estimer la contribution complémentaire, nous utilisons les relations (1.17), (1.18) et (1.19) pour une suite majorant $\{|f(n)|\}_{n=1}^{\infty}$ et dont la série de Dirichlet appartient à $\mathcal{E}_{\kappa_{0}}(\beta, c, \delta)$ ou $\mathcal{E}_{\kappa_{0}}^{*}(\delta)$. Posant

$$
D(t):= \begin{cases}L_{\beta+\delta / 2}(t) & \text { si } f \in \mathcal{H}\left(\kappa, \kappa_{0} ; \beta, c, \delta\right), \\ t^{\delta /\left(v_{f}+2\right)} & \text { si } f \in \mathcal{H}^{*}\left(\kappa, \kappa_{0} ; \delta\right),\end{cases}
$$

cela fournit

$$
\sum_{t \leqslant n \leqslant t+z}|f(n)| \ll z(\log t)^{\kappa_{0}-1}+t / D(t) \quad(t \geqslant 2,1 \leqslant z \leqslant t) .
$$


Nous en déduisons que, pour tout entier $m$ de $[0, T]$,

$$
\sum_{m / T \leqslant|\log (N / n)|<(m+1) / T} \frac{|f(n)|}{n^{\alpha+\sigma}} \ll \frac{(\log N)^{\kappa_{0}-1}}{(m+1) T}+\frac{1}{(m+1) D(N)} .
$$

Par sommation sur $m$, nous obtenons ainsi que, sous l'hypothèse $N>|\tau|^{1 / 2}$,

$$
R(N, T) \ll N^{1-\sigma}\left(\frac{(\log N)^{\kappa_{0}}}{T}+\frac{\log N}{D(N)}\right) .
$$

Montrons l'assertion (i). Nous évaluons l'intégrale de (4·6) en déplaçant l'abscisse d'intégration vers la gauche jusqu'à $\Re e w=1-\sigma-\eta$ avec

$$
\eta:=c /(\log |\tau|)^{(1-\beta) / \beta},
$$

et nous appliquons le théorème des résidus. Il s'ensuit que

$$
\frac{1}{2 \pi i} \int_{\alpha-i T}^{\alpha+i T} \mathcal{F}(w+s) \frac{N^{w}}{w} \mathrm{~d} w=\mathcal{F}(s)+\frac{1}{2 \pi i} \int_{\mathcal{L}} \mathcal{F}(w+s) \frac{N^{w}}{w} \mathrm{~d} w,
$$

où $\mathcal{L}$ est la ligne brisée joignant les points $\alpha-i T, 1-\sigma-\eta-i T, 1-\sigma-\eta+i T$, $\alpha+i T$. La majoration classique de $\zeta_{\mathbb{K}}(s)$ dans le domaine de Vinogradov et l'hypothèse $(1 \cdot 7)$ fournissent alors

$$
\int_{\mathcal{L}} \mathcal{F}(w+s) \frac{N^{w}}{w} \mathrm{~d} w \ll N^{1-\sigma}\left(\frac{|\tau|^{1-\delta / 2}}{N^{\eta}}+\frac{1}{|\tau|^{\delta / 2}}\right) .
$$

Le résultat de la première assertion découle immédiatement de (4·6), (4.8), (4.9) et (4.10) pour les valeurs indiquées de $N$.

La démonstration de l'assertion (ii) est similaire. Nous évaluons à présent l'intégrale de $(4 \cdot 6)$, en choisissant $\eta:=\delta /\left(v_{f}+2\right)$. La borne de convexité

$$
\zeta_{\mathbb{K}}(a+i b) \ll_{a}|b|^{[\mathbb{K}: \mathbb{Q}](1-a) / 2} \quad\left(\frac{1}{2} \leqslant a<1 \leqslant|b|\right)
$$

valable pour tout corps de nombres $\mathbb{K}$ et l'hypothèse $(1 \cdot 7)$ fournissent alors

$$
\mathcal{F}(s+w) \ll T^{v_{f} \delta /\left(v_{f}+2\right)+1-\delta}=T^{1-2 \delta /\left(v_{f}+2\right)} \quad(w \in \mathcal{L})
$$

et

$$
\begin{aligned}
\int_{\mathcal{L}} \mathcal{F}(w+s) \frac{N^{w}}{w} \mathrm{~d} w & \ll N^{1-\sigma}\left(\frac{1}{T^{2 \delta /\left(v_{f}+2\right)}}+\frac{T^{1-2 \delta /\left(v_{f}+2\right)} \log T}{N^{\delta /\left(v_{f}+2\right)}}\right) \\
& \ll \frac{N^{1-\sigma} \log N}{T^{2 \delta /\left(v_{f}+2\right)}},
\end{aligned}
$$

dès que $N>T^{\left(v_{f}+2\right) / \delta}$. Comme $2 \delta /\left(v_{f}+2\right) \leqslant 1$, le résultat annoncé découle immédiatement de $(4 \cdot 6),(4 \cdot 8),(4 \cdot 9)$ et $(4 \cdot 11)$. 
Lemme 4.5. Soient $\beta, c, \delta, \kappa$, des nombres réels positifs tels que $\beta+\delta<3 / 5$, et $f \in \mathcal{H}_{+}(\kappa ; \beta, c, \delta)$. Notant $T:=L_{\beta+\delta / 2}(y)$, nous avons

$$
\frac{1}{2 \pi i} \int_{\alpha_{0}-i T}^{\alpha_{0}+i T} \mathcal{F}(s ; y) \frac{x^{s}}{s} \mathrm{~d} s=\left\{1+O\left(\frac{1}{L_{\beta}(y)}\right)\right\} \Lambda_{f}(x, y)+O\left(\frac{x}{L_{\beta+\delta / 3}(x)}\right)
$$

uniformément pour $(x, y)$ dans le domaine $\left(G_{\beta}\right)$.

De plus, si $\kappa \in \mathbb{N}^{*}$ et $f \in \mathcal{H}_{+}^{*}(\kappa ; \beta, c, \delta)$, la formule (4.12) est valable dans le domaine $\left(H_{\beta}\right)$ et le second terme d'erreur peut être supprimé.

Démonstration. Appliquons le Lemme 4.1 avec $(\beta+\delta / 2, \delta / 2)$ au lieu de $(\beta, \delta)$. Nous obtenons, avec la notation (4.3),

$$
\int_{\alpha_{0}-i T}^{\alpha_{0}+i T} \mathcal{F}(s ; y) \frac{x^{s}}{s} \mathrm{~d} s=(\log y)^{\kappa} \int_{\alpha_{0}-i T}^{\alpha_{0}+i T} J_{f}(s) \widehat{\varrho_{\kappa}}((s-1) \log y) x^{s} \mathrm{~d} s+R
$$

avec

$$
R \ll \frac{x^{\alpha_{0}} \mathcal{F}\left(\alpha_{0} ; y\right) \log T}{T} \ll \frac{x \varrho_{\kappa}(u)}{L_{\beta}(y)} .
$$

Soit $V:=L_{\beta+\delta / 2}(x)$. Dans un premier temps, établissons que l'on peut étendre au segment $\left[\alpha_{0}-i V, \alpha_{0}+i V\right]$ l'intégrale figurant au membre de droite de (4.13) moyennent une erreur englobée par celle de $(4 \cdot 12)$.

Les bornes de convexité pour les fonctions $\zeta_{\mathbb{K}}(s)$ et l'hypothèse (1.7) fournissent

$$
\mathcal{F}(s) \ll|\tau|^{1-\delta / 2} \quad\left(\sigma \geqslant \alpha_{0}>1-\delta, T \leqslant|\tau| \leqslant V\right) .
$$

De plus, d'après le lemme III.5.8.2 de [16], nous avons

$(4 \cdot 15)(s-1)^{\kappa}(\log y)^{\kappa} \widehat{\varrho_{\kappa}}((s-1) \log y)=1+O\left(\frac{1+u \xi_{\kappa}(u)}{|\tau| \log y}\right) \quad\left(\sigma=\alpha_{0},|\tau| \geqslant T\right)$.

Nous avons donc

$$
(\log y)^{\kappa} \int_{\substack{\sigma=\alpha_{0} \\ T \leqslant|\tau| \leqslant V}} J_{f}(s) \widehat{\varrho_{\kappa}}((s-1) \log y) x^{s} \mathrm{~d} s=\int_{\substack{\sigma=\alpha_{0} \\ T \leqslant|\tau| \leqslant V}} \mathcal{F}(s) \frac{x^{s}}{s} \mathrm{~d} s+E
$$

avec

$$
E \ll x^{\alpha_{0}} \int_{T}^{V} \frac{1+u \xi_{\kappa}(u)}{\tau^{1+\delta / 2} \log y} \mathrm{~d} \tau \ll \frac{x^{\alpha_{0}}\left(1+u \xi_{\kappa}(u)\right)}{T^{\delta / 2}} \ll \frac{x \varrho_{\kappa}(u)}{L_{\beta}(y)} .
$$

Appliquant le Lemme 4.4(i) pour le choix $(\beta+\delta / 2, \delta / 2)$ avec

$$
N=N_{\tau}:=\exp \left\{(1 / c)(\log |\tau|)^{1 /(\beta+\delta / 2)}\right\},
$$

nous avons en outre

$$
\begin{aligned}
\int_{\substack{\sigma=\alpha_{0} \\
T \leqslant|\tau| \leqslant V}} \mathcal{F}(s) \frac{x^{s}}{s} \mathrm{~d} s= & \sum_{n \leqslant x^{1 / c}} f(n) \int_{T_{n} \leqslant|\tau| \leqslant V}\left(\frac{x}{n}\right)^{s} \frac{\mathrm{d} s}{s} \\
& +O\left(x^{\alpha_{0}} \int_{T}^{V} \frac{N_{\tau}^{1-\alpha_{0}}\left(\log N_{\tau}\right)^{\kappa_{0}+1}}{\tau^{1+\delta / 2}} \mathrm{~d} \tau\right),
\end{aligned}
$$

où l'on a posé $T_{n}:=\max \left\{L_{\beta+\delta / 2}\left(n^{c}\right), T\right\}$. 
Lorsque $(x, y) \in G_{\beta}$, nous avons $u \leqslant(\log y)^{\beta /(1-\beta)}$, d'où

$$
\log |\tau| \leqslant(u \log y)^{\beta+\delta / 2} \leqslant(\log y)^{(\beta+\delta / 2) /(1-\beta)} \quad(|\tau| \leqslant V)
$$

et donc

$$
N_{\tau}^{1-\alpha_{0}}\left(\log N_{\tau}\right)^{\kappa_{0}+1} \ll|\tau|^{\delta / 4} \quad(|\tau| \leqslant V) .
$$

Le terme d'erreur de $(4 \cdot 18)$ est donc

$$
\ll \frac{x^{\alpha_{0}}}{T^{\delta / 4}} \ll \frac{x \varrho_{\kappa}(u)}{L_{\beta}(y)} .
$$

En vertu de la formule (II·2·7) de [16], dont (3·13) est une variante, le terme général de la somme en $n$ de $(4 \cdot 18)$ est

$$
\ll\left(\frac{x}{n}\right)^{\alpha_{0}} \frac{f(n)}{1+T_{n}|\log (x / n)|} .
$$

Désignons par $S_{1}$ la contribution des entiers $n$ tels que $|\log (x / n)| \leqslant L_{\beta+\delta / 2}\left(n^{c}\right)^{-1 / 2}$ et par $S_{2}$ la contribution complémentaire. Comme tout entier $n$ compté dans $S_{1}$ vérifie $|x-n| \ll x / \sqrt{V}$, nous avons, grâce à (4.7),

$$
S_{1} \ll \sum_{|x-n| \leqslant x / \sqrt{V}} f(n) \ll x / V^{1 / 4} .
$$

Par ailleurs, nous avons

$$
\begin{aligned}
S_{2} & \leqslant \sum_{\substack{n \leqslant x^{1 / c} \\
|\log (x / n)|>L_{\beta+\delta / 2}\left(n^{c}\right)^{-1 / 2}}} \frac{f(n)(x / n)^{\alpha_{0}}}{1+\left(L_{\beta+\delta / 2}\left(n^{c}\right)+T\right)|\log (x / n)|} \\
& \ll x^{\alpha_{0}} \sum_{n \leqslant x^{1 / c}} \frac{f(n) / n^{\alpha_{0}}}{L_{\beta+\delta / 2}\left(n^{c}\right)^{1 / 2}+T L_{\beta+\delta / 2}\left(n^{c}\right)^{-1 / 2}} \\
& \ll x^{\alpha_{0}}\left(\frac{u \xi_{\kappa}(u)+1}{T^{1 / 2}} \sum_{n \leqslant y} \frac{f(n)}{n}+\sum_{y<n \leqslant x^{1 / c}} \frac{f(n)}{n L_{\beta+\delta / 2}\left(n^{c}\right)^{1 / 4}}\right) \\
& \ll \frac{x^{\alpha_{0}}}{L_{\beta+\delta / 2}(y)^{c_{6}}} \ll \frac{x \varrho_{\kappa}(u)}{L_{\beta}(y)},
\end{aligned}
$$

où $c_{6}$ est une constante positive convenable. Nous avons donc établi que

$$
(\log y)^{\kappa} \int_{\substack{\sigma=\alpha_{0} \\ T \leqslant|\tau| \leqslant V}} J_{f}(s) \widehat{\varrho_{\kappa}}((s-1) \log y) x^{s} \mathrm{~d} s \ll \frac{x}{V^{1 / 4}}+\frac{x \varrho_{\kappa}(u)}{L_{\beta}(y)},
$$


et donc, en reportant dans $(4 \cdot 13)$,

$$
\begin{aligned}
\int_{\alpha_{0}-i T}^{\alpha_{0}+i T} \mathcal{F}(s ; y) \frac{x^{s}}{s} \mathrm{~d} s= & (\log y)^{\kappa} \int_{\alpha_{0}-i V}^{\alpha_{0}+i V} J_{f}(s) \widehat{\varrho_{\kappa}}((s-1) \log y) x^{s} \mathrm{~d} s \\
& +O\left(\frac{x}{V^{1 / 4}}+\frac{x \varrho_{\kappa}(u)}{L_{\beta}(y)}\right) .
\end{aligned}
$$

Déplaçons ensuite l'abscisse d'intégration vers la droite jusqu'à $\sigma_{x}:=1+1 / \log x$. Les intégrales sur les segments $\left[\alpha_{0} \pm i V, \sigma_{x} \pm i V\right]$ étant estimées grâce à (4.14) et $(4 \cdot 15)$, nous obtenons

$$
\begin{aligned}
\frac{1}{2 \pi i} \int_{\alpha_{0}-i T}^{\alpha_{0}+i T} \mathcal{F}(s ; y) \frac{x^{s}}{s} \mathrm{~d} s= & \frac{(\log y)^{\kappa}}{2 \pi i} \int_{\sigma_{x}-i V}^{\sigma_{x}+i V} J_{f}(s) \widehat{\varrho_{\kappa}}((s-1) \log y) x^{s} \mathrm{~d} s \\
& +O\left(\frac{x}{V^{\delta / 4}}+\frac{x \varrho_{\kappa}(u)}{L_{\beta}(y)}\right) .
\end{aligned}
$$

Étendons à présent à la droite $\sigma=\sigma_{x}$ tout entière le domaine d'intégration de l'intégrale du membre de droite et estimons l'erreur commise en appliquant (4.15), puis (4.14) (pour prendre en compte le terme d'erreur de (4.15)), et enfin une formule version effective de la formule de Perron, comme celle du corollaire II.2.2.1 de [16], pour traiter le terme principal. On vérifie sans peine que cela n'altère pas le terme résiduel.

En utilisant les deux premières relations (3.9), le théorème de convolution et le théorème d'inversion de Laplace, on voit ensuite que l'intégrale étendue coïncide avec le terme principal de $(4 \cdot 12)$. Cela établit la première assertion de l'énoncé.

Quand $\kappa \in \mathbb{N}^{*}$ et $f \in \mathcal{H}_{+}^{*}(\kappa ; \beta, c, \delta)$, la fonction $J_{f}(s)$ est holomorphe dans le demi-plan $\sigma \geqslant 1-\delta$. Nous pouvons donc écrire, sous réserve de convergence,

$$
\frac{(\log y)^{\kappa}}{2 \pi i} \int_{\alpha_{0}-i \infty}^{\alpha_{0}+i \infty} J_{f}(s) \widehat{\varrho_{\kappa}}((s-1) \log y) x^{s} \mathrm{~d} s=\Lambda_{f}(x, y) .
$$

Compte tenu de la validité de $(1 \cdot 24)$ dans le domaine $\left(H_{\beta}\right)$, il ne reste donc à montrer que l'estimation

$$
(\log y)^{\kappa} \int_{\substack{\sigma=\alpha_{0} \\|\tau| \geqslant T}} J_{f}(s) \widehat{\varrho_{\kappa}}((s-1) \log y) x^{s} \mathrm{~d} s \ll \frac{x \varrho_{\kappa}(u)}{L_{\beta}(y)} .
$$

À cette fin, nous utilisons, comme précédemment, (4·14) et (4·15). La contribution au membre de droite de (4.19) du terme d'erreur de (4.15) est

$$
\ll x^{\alpha_{0}} \int_{T}^{\infty} \frac{1+u \xi_{\kappa}(u)}{\tau^{1+\delta / 2} \log y} \mathrm{~d} \tau \ll \frac{x^{\alpha_{0}}\left(1+u \xi_{\kappa}(u)\right)}{T^{\delta / 2}} \ll \frac{x \varrho_{\kappa}(u)}{L_{\beta}(y)} .
$$

Pour estimer celle du terme principal, nous employons le Lemme 4.4(ii) avec $\sigma=\alpha_{0}$ et $N=N_{\tau}=|\tau|^{\left(v_{f}+2\right) / \delta}$. Nous obtenons ainsi

$$
\int_{\substack{\sigma=\alpha_{0} \\|\tau| \geqslant T}} \mathcal{F}(s) \frac{x^{s}}{s} \mathrm{~d} s=\sum_{n \geqslant 1} f(n) \int_{|\tau| \geqslant T_{n}}\left(\frac{x}{n}\right)^{s} \frac{\mathrm{d} s}{s}+O\left(\frac{x^{\alpha_{0}}}{L_{\beta}(y)}\right),
$$

où l'on a posé $T_{n}:=\max \left\{n^{\delta /\left(v_{f}+2\right)}, T\right\}$. 
Le terme résiduel de $(4 \cdot 21)$ est pleinement acceptable. Le terme général de la somme en $n$ est

$$
\ll\left(\frac{x}{n}\right)^{\alpha_{0}} \frac{1}{1+T_{n}|\log (x / n)|} .
$$

Désignons par $S_{1}^{*}$ la contribution des entiers $n$ satisfaisant $|x-n| \leqslant x^{1-\delta /\left(2 v_{f}+4\right)}$ et par $S_{2}^{*}$ la contribution complémentaire. Nous avons, grâce à $(4 \cdot 7)$,

$$
S_{1}^{*} \ll \sum_{|x-n| \leqslant x^{1-\delta /\left(2 v_{f}+4\right)}} f(n) \ll x^{1-\delta /\left(2 v_{f}+4\right)}(\log x)^{\kappa_{0}} .
$$

Par ailleurs, comme $\alpha_{0} \geqslant 1-\delta /\left(4 v_{f}+8\right)$ dans le domaine $\left(H_{\beta}\right)$ pour $y$ assez grand, chaque entier $n$ compté dans $S_{2}^{*}$ vérifie sous cette hypothèse

$$
\begin{aligned}
n^{\alpha_{0}} T_{n}|\log (x / n)| & \geqslant n^{1-\delta /\left(4 v_{f}+8\right)} n^{\delta /\left(2 v_{f}+4\right)} T^{1 / 2}|\log (x / n)| \\
& \gg n^{1+\delta /\left(4 v_{f}+8\right)} T^{1 / 2} .
\end{aligned}
$$

Il suit

$$
S_{2}^{*} \ll x^{\alpha_{0}} \sum_{n \geqslant 1} \frac{f(n)}{n^{1+\delta /\left(4 v_{f}+8\right)} T^{1 / 2}} \ll \frac{x^{\alpha_{0}}}{L_{\beta}(y)} .
$$

Cette estimation étant trivialement réalisée lorsque $y$, et donc $x$, est borné, cela établit bien (4·19) et achève ainsi la démonstration.

\subsection{Preuve du Théorème 1.2}

Soit $T:=L_{\beta+\delta / 2}(y)$. La formule de Perron effective assortie à l'estimation dans les petits intervalles du Lemme 4.3 permet aisément de montrer que l'on a

$$
M\left(x ; f_{y}\right)=\frac{1}{2 \pi i} \int_{\alpha_{0}-i T}^{\alpha_{0}+i T} \mathcal{F}(s ; y) \frac{x^{s}}{s} \mathrm{~d} s+O\left(\frac{x \varrho_{\kappa}(u)}{L_{\beta}(y)}\right)
$$

uniformément pour $(x, y) \in\left(H_{\beta}\right)$. Compte tenu de la seconde assertion du Lemme 4.5 , cela implique immédiatement le résultat annoncé lorsque $\kappa \in \mathbb{N}^{*}$, $f \in \mathcal{H}_{+}^{*}(\kappa ; \beta, c, \delta)$.

La première assertion est obtenue en observant que, sous la condition $\left(G_{\beta}\right)$, on a

$$
\frac{1}{L_{\beta+\delta / 3}(x)} \ll \frac{\varrho_{\kappa}(u)}{L_{\beta}(y)},
$$

pourvu que la constante $A$ soit convenablement choisie. 


\section{Fonctions zêtas de Dedekind généralisées}

Soit $F$ un polynôme à coefficients entiers, sans facteur carré, de degré $d$. Notant $\mathcal{P}$ l'ensemble des nombres premiers, on définit $\varrho_{F}(p): \mathcal{P} \rightarrow[0, d]$ comme le nombre de racines de $F$ modulo $p$, comptées avec multiplicité. Dans la suite, on notera $\mathbb{L}=\mathbb{L}_{F}$ le corps de décomposition de $F, \zeta_{\mathbb{L}}$ sa fonction zêta de Dedekind, et $G$ le groupe de Galois de $\mathbb{L} / \mathbb{Q}$.

Un élément $\gamma$ de $G$ permute les racines de $F$; nous notons $n_{\gamma}$ le nombre de points fixes de cette permutation. Ce nombre est invariant par conjugaison, et cette action réalise $G$ comme un sous-groupe du groupe symétrique $S_{d}$.

L'objet de ce paragraphe consiste à établir le résultat suivant, qui complète la preuve du Théorème 2.4. Étant donnée une fonction arithmétique totalement multiplicative $\mathcal{J}: \mathbb{N} \rightarrow \mathbb{R}^{+}$, nous posons

$$
\mathcal{F}_{F, \mathcal{J}}(s):=\sum_{n \geqslant 1} \frac{\mathcal{J}\left(\varrho_{F}(n)\right)}{n^{s}} \quad(\sigma>1)
$$

et

$$
\kappa_{F, \mathfrak{J}}:=\frac{1}{|G|} \sum_{\gamma \in G} \mathcal{J}\left(n_{\gamma}\right)
$$

Proposition 5.1. Pour tous $\beta>0, \delta>0$, tels que $\beta+\delta<3 / 5$, il existe une constante $c>0$ telle que la fonction $H$ définie dans le demi-plan $\sigma>1$ par la relation

$$
\mathcal{F}_{F, \mathcal{J}}(s)=\zeta(s)^{\kappa_{F, \mathcal{J}}} H(s),
$$

soit prolongeable en une fonction holomorphe et sans zéro dans le domaine (1.6), vérifiant les conditions $(1 \cdot 7)$ et $(1 \cdot 10)$.

Comme $\mathcal{J}$ o $\varrho_{F}$ est multiplicative et comme $F$ est sans facteur carré, le facteur eulérien associé à $p$ est, sauf pour un nombre fini de valeurs de $p$, une fonction holomorphe de $s$ de la forme

$$
\exp \left\{\partial\left(\varrho_{F}(p)\right) / p^{s}+O\left(1 / p^{2 \sigma}\right)\right\}
$$

De plus, lorsque $p$ est borné, les facteurs locaux

$$
\sum_{\nu \geqslant 0} \frac{\partial\left(\varrho_{F}\left(p^{\nu}\right)\right)}{p^{\nu s}}
$$

définissent des fonctions holomorphes pour $\sigma>0$ : cela découle immédiatement du fait que $\varrho_{F}\left(p^{\nu}\right) \ll 1$ pour tous $p$ et $\nu$. Nous pouvons donc nous restreindre à prouver les assertions de l'énoncé pour le produit eulérien

$$
\exp \left\{\sum_{p} \mathcal{J}\left(\varrho_{F}(p)\right) / p^{s}\right\}
$$


Étant donnés un nombre premier $p$ non ramifié dans $\mathbb{L}$ et un idéal premier $\mathfrak{P}$ de $\mathbb{L}$ divisant $p$, considérons le groupe de décomposition $D(\mathfrak{P})$ de $\mathfrak{P}$, c'est-àdire le sous-groupe de $G$ constitué des automorphismes $\gamma$ tels que $\gamma(\mathfrak{P})=\mathfrak{P}$. L'application naturelle $D(\mathfrak{P}) \rightarrow \operatorname{Gal}\left(\left(\mathbb{Z}_{\mathbb{L}} / \mathfrak{P}\right) /(\mathbb{Z} / p \mathbb{Z})\right)$ est alors un isomorphisme. Notons $(\mathfrak{P}, \mathbb{L} / \mathbb{Q})$ l'antécédent de l'application de Frobenius $x \mapsto x^{p}$ par cet isomorphisme. Le symbole d'Artin de $p$ relativement à l'extension $\mathbb{L} / \mathbb{Q}$, noté $(p, \mathbb{L} / \mathbb{Q})$, est alors défini comme l'ensemble

$$
\left\{(\mathfrak{P}, \mathbb{L} / \mathbb{Q}): \mathfrak{P} \mid p \mathbb{Z}_{L}\right\} .
$$

C'est une classe de conjugaison de $G$, qui agit sur les racines de $F$.

La caractérisation suivante de $\varrho_{F}(p)$ est classique.

Lemme 5.2. Pour tout nombre premier $p$ assez grand, $\varrho_{F}(p)$ est égal au nombre de points fixes du symbole d'Artin $(p, \mathbb{L} / \mathbb{Q})$ agissant sur les racines de $F$.

Démonstration. Observons tout d'abord que ce nombre de points fixes ne dépend pas de l'élément choisi dans la classe de conjugaison $(p, \mathbb{L} / \mathbb{Q})$.

Soit $\mathfrak{P}$ un idéal premier de l'anneau $\mathbb{Z}_{\mathbb{L}}$ des entiers $\mathbb{L}$, contenant $p$. On sait alors que les racines de $F$ modulo $p$ sont exactement les racines de $F$ dans $\mathbb{Z}_{\mathbb{L}} / \mathfrak{P}$ qui sont fixées par $\operatorname{Gal}\left(\left(\mathbb{Z}_{\mathbb{L}} / \mathfrak{P}\right) /(\mathbb{Z} / p \mathbb{Z})\right)$.

En d'autres termes, $\varrho_{F}(p)$ est le nombre de points fixes de l'application de Frobenius $x \mapsto x^{p}$ agissant sur les racines de $F$ dans $\mathbb{Z}_{\mathbb{L}} / \mathfrak{P}$; mais si $p$ est non ramifié dans $\mathbb{L} / \mathbb{Q}$, cette action coïncide avec l'action de $(\mathfrak{P}, \mathbb{L} / \mathbb{Q})$ sur les racines de $F$ dans $\mathbb{L}$. Comme $(\mathfrak{P}, \mathbb{L} / \mathbb{Q})$ appartient à la classe de conjugaison $(p, \mathbb{L} / \mathbb{Q})$, cela conclut la preuve.

Introduisons à présent l'ensemble $X$ des caractères irréductibles de $G$, et l'ensemble $\mathcal{C}$ des classes de conjugaison de $G$. Un caractère étant constant sur une classe de conjugaison, l'expression $\chi(C)$ pour $(\chi, C) \in X \times \mathcal{C}$ a bien un sens.

Lemme 5.3. Pour tous $\beta>0, \delta>0$, tels que $\beta+\delta<3 / 5$, il existe une constante $c>0$ telle que, pour tout $s$ de la région (1.6), il existe un nombre fini de sousextensions $\mathbb{L}_{i}$ de $\mathbb{L}$, des caractères de Hecke $\psi_{i j}$ non principaux de $\mathbb{L}_{i}$, et des nombres complexes $\kappa_{i j}$ tels que

$$
\mathcal{F}_{F, \mathcal{J}}(s)=\zeta(s)^{\kappa_{F, \mathcal{J}}} G_{0}(s) \prod_{i, j} L\left(s, \psi_{i j}\right)^{\kappa_{i j}},
$$

où $G_{0}$ est une série de Dirichlet absolument convergente et sans zéro pour $\sigma>1 / 2$.

Démonstration. D'après les formules d'orthogonalité des caractères, on a, pour toute classe de conjugaison $C$ de $G$,

$$
\mathbf{1}_{C}(g)=\frac{|C|}{|G|} \sum_{\chi \in X} \bar{\chi}(C) \chi(g) .
$$


En particulier,

$$
\begin{aligned}
\mathcal{J}\left(\varrho_{F}(p)\right) & =\sum_{C \in \mathcal{C}} \frac{\mathcal{J}(N(C))|C|}{|G|} \sum_{\chi \in X} \bar{\chi}(C) \chi((p, \mathbb{L} / \mathbb{Q})) \\
& =\frac{1}{|G|} \sum_{\gamma \in G} \sum_{\chi \in X} \mathcal{J}\left(n_{\gamma}\right) \bar{\chi}(\gamma) \chi((p, \mathbb{L} / \mathbb{Q})) .
\end{aligned}
$$

Reportons dans $(5 \cdot 1)$ en tenant compte de la réduction du cas général à ce cas particulier. Nous obtenons immédiatement la formule suivante, faisant intervenir les fonctions $L$ d'Artin

$$
\mathcal{F}_{F, \mathcal{I}}(s)=\zeta(s)^{\kappa_{F, \mathcal{J}}} \prod_{(\gamma, \chi) \in G \times X}^{*} L(s, \chi, \mathbb{L} / \mathbb{Q})^{\alpha_{F, \mathcal{J}, \chi}} G_{0}(s),
$$

où $G_{0}(s)$ est une série de Dirichlet absolument convergente et non nulle dans le demi-plan $\sigma>1 / 2$, et où l'astérisque indique que le produit sur les $\chi$ exclut le caractère trivial. Cette identité est valide dans toute région dans laquelle les fonctions $L$ d'Artin y apparaissant n'ont ni zéro ni pôle.

D'après le théorème de Brauer [2], toute fonction $L$ d'Artin associée à un caractère irréductible non trivial est un produit fini de puissances entières, positives ou négatives, de fonctions $L(s, \psi)$ de Hecke associées à des sous-extensions de $\mathbb{L}$ et correspondant à des caractères $\psi$ non principaux. De plus, toute région sans zéro pour $\zeta_{\mathbb{L}}$ est également une région sans zéro pour les $L(s, \psi)$. Comme il est établi dans [7] que les fonctions zêtas de Dedekind ont, aux constantes multiplicatives près, les mêmes régions sans zéro que la fonction zêta de Riemann, nous obtenons bien la conclusion requise.

Remarque. On peut également utiliser la décomposition explicite, due à Deuring [3], de la fonction $\mathbf{1}_{C}$ en somme de caractères induits par des caractères monomiaux; cela permet, en cas de nécessité, de contrôler les exposants $\kappa_{i, j}$.

Nous supposons dans toute la suite que la constante $c$ est choisie de telle sorte que la formule (5.2) soit valide dans la région (1·6). La condition (1.7) découle alors directement du lemme suivant :

Lemme 5.4. Dans la région (1.6), on a pour tout caractère de Hecke $\psi$ non principal,

$$
|\log L(s, \psi)| \leqslant \frac{1-\beta-\delta}{\beta+\delta} \log _{2}(3+|\tau|)+O(1) .
$$

Démonstration. L'identité est immédiate pour $\sigma>1$, où l'on dispose de l'estimation $1 \ll L(s, \psi) \ll \zeta_{\mathbb{L}}(\sigma)$.

Dans le demi-plan $\sigma \leqslant 1$, l'existence d'une région sans zéro associée à une formule explicite telle la formule (5.9) de [6] fournit classiquement la majoration 


$$
\left|\frac{L^{\prime}(s, \psi)}{L(s, \psi)}\right| \ll\{\log (3+|\tau|)\}^{(1-\beta-\delta) /(\beta+\delta)} .
$$

En intégrant cette majoration sur le segment

$$
\left[1+1 /\left\{\log (3+|\tau|\}^{(1-\beta-\delta) /(\beta+\delta)}+i \tau, s\right],\right.
$$

nous obtenons bien le résultat annoncé.

La condition (1.10) peut être obtenue de façon très voisine de celle du lemme 6.3 de [4]; grâce au Lemme 5.3, nous pouvons nous ramener au cas d'une seule fonction $L$. Nous nous contentons d'indiquer les grandes lignes de la preuve.

Lemme 5.5. Soit $\psi$ un caractère de Hecke non principal pour l'extension $\mathbb{L} / \mathbb{Q}$. Pour tous $\beta>0, \delta>0$, tels que $\beta+\delta<3 / 5$, il existe une constante $c$ telle que, dans la région $(1 \cdot 11)$, on ait

$$
L(s, \psi ; y):=\prod_{N \mathfrak{P} \leqslant y}\left(1-\psi(\mathfrak{P}) /(N \mathfrak{P})^{s}\right)^{-1}=L(s, \psi)+O\left(\frac{1}{L_{\beta+\delta}(y)^{2}}\right) .
$$

Démonstration. Commençons par diminuer, si nécessaire, la valeur de $c$ de façon à ce que l'on ait, pour tous $t \geqslant y \geqslant 2$,

$$
\sum_{N \mathfrak{a} \leqslant t} \psi(\mathfrak{a}) \Lambda(\mathfrak{a}) \ll t / L_{\beta+\delta}(y)^{c},
$$

où la somme porte sur les idéaux entiers $\mathfrak{a}$ de $\mathbb{L}$ dont la norme $N \mathfrak{a}$ n'excède pas $t$.

Remarquons ensuite que

$$
-\frac{L^{\prime}(s, \psi ; y)}{L(s, \psi ; y)}=\sum_{N \mathfrak{a} \leqslant y} \frac{\psi(\mathfrak{a}) \Lambda(\mathfrak{a})}{(N \mathfrak{a})^{s}}+O\left(y^{1 / 2-\sigma}\right) .
$$

Dans le cas où $\sigma \geqslant 1+6 /(\log y)^{1-\beta-\delta}$, une sommation d'Abel utilisant (5.5) conduit à

$$
-\frac{L^{\prime}(s, \psi)}{L(s, \psi)}=\sum_{N \mathfrak{a} \leqslant y} \frac{\psi(\mathfrak{a}) \Lambda(\mathfrak{a})}{(N \mathfrak{a})^{s}}+O\left(y^{1-\sigma} L_{\beta+\delta}(y)^{-c}\right),
$$

où le terme reste peut être remplacé par $O\left(y^{(1-\sigma) / 2} L_{\beta+\delta}(y)^{-3-c}\right)$.

Dans le cas contraire, posons $\sigma_{0}:=1-\sigma+6 /(\log y)^{1-\beta-\delta}>0$ et $T:=L_{\beta+\delta}(y)^{9}$. La formule de Perron effective permet d'écrire

$$
\sum_{N \mathfrak{a} \leqslant y} \frac{\psi(\mathfrak{a}) \Lambda(\mathfrak{a})}{(N \mathfrak{a})^{s}}=\frac{-1}{2 \pi i} \int_{\sigma_{0}-i T}^{\sigma_{0}+i T} \frac{L^{\prime}(s+w, \psi)}{L(s+w, \psi)} \frac{y^{w}}{w} \mathrm{~d} w+O\left(\frac{y^{1-\sigma}(\log y)^{1+\beta+\delta}}{L_{\beta+\delta}(y)^{3}}\right) .
$$


Déplaçons l'abscisse d'intégration jusqu'à $\sigma_{1}:=-1 /(\log y)^{1-\beta-\delta}$. Quitte à diminuer encore la valeur de $c$, la région d'intégration reste incluse dans la région sans zéro de la fonction $L$. En particulier, la seule singularité de l'intégrande traversée est $w=0$, qui contribue pour $-L^{\prime}(s, \psi) / L(s, \psi)$.

D'après le Lemme 5.4, la contribution des parties horizontales est

$$
\ll \frac{y^{1-\sigma}(\log y)^{1-\beta-\delta}}{L_{\beta+\delta}(y)^{3}}
$$

alors que celle du segment déplacé vaut

$$
\frac{-1}{2 \pi i} \int_{\sigma_{1}-i T}^{\sigma_{1}+i T} \frac{L^{\prime}(s+w, \chi) y^{w}}{L(s+w, \chi) w} \mathrm{~d} w \ll y^{\sigma_{1}} \log y .
$$

Nous obtenons donc

$$
\frac{L^{\prime}(s, \psi ; y)}{L(s, \psi ; y)}-\frac{L^{\prime}(s, \psi)}{L(s, \psi)} \ll \frac{y^{1-\sigma}(\log y)^{1+\beta+\delta}}{L_{\beta+\delta}(y)^{3}} .
$$

En intégrant cette majoration sur la demi-droite $[s,+\infty+i \tau]$, il suit

$$
L(s, \psi ; y)=L(s, \psi)\left\{1+O\left(\frac{(\log y)^{\beta+\delta}}{L_{\beta+\delta}(y)^{3-c}}\right)\right\} .
$$

La relation $(5 \cdot 4)$ en découle immédiatement, quitte à imposer $c<1$.

\section{Bibliographie}

[1] M. Balazard \& G. Tenenbaum, Sur la répartition des valeurs de la fonction d'Euler, Compositio Math. 110 (1998), no. 2, 239-250.

[2] R. Brauer, On Artin's L-series with general group characters, Ann. of Math. 48 (1947), $502-514$.

[3] M. Deuring, Über den Tschebotareffschen Dichtigkeitsatz, Math. Ann. 110 (1934), 414415.

[4] É. Fouvry \& G. Tenenbaum, Entiers sans grands facteurs premiers en progression arithmétique, Proc. London Math. Soc. 63 (1991), 449-494.

[5] A. Hildebrand \& G. Tenenbaum, On a class of difference differential equations arising in number theory, J. d'Analyse 61 (1993), 145-179.

[6] J. Lagarias \& A. Odlyzko, Effective versions of the Chebotarev density theorem, in A. Fröhlich (ed.), Algebraic Number Fields (L-functions and Galois properties), Academic Press (1977)

[7] T. Mitsui, On the prime ideal theorem, J. Math. Soc. Japan 20 (1968), 233-247.

[8] M. Naïmi, Répartition des valeurs de la fonction $\varphi$ d'Euler et de la fonction somme des diviseurs sur les entiers sans grand facteur premier, Ann. Univ. Sci. Budapest. Eötvős Sect. Math. 42 (1999), 147-164 (2000).

[9] H. Osada, The Galois groups of the polynomials $X^{n}+a X+b$, J. Number Theory 25 (1987), 230-238. 
[10] E. Saias, Sur le nombre des entiers sans grand facteur premier, J. Number Theory 32 (1989), 78-99.

[11] E.J. Scourfield, On ideals free of large prime factors, J. de Théorie des Nombres de Bordeaux 16 (2004), 733-772.

[12] A. Smati \& J. Wu, Distribution of values of some multiplicative functions over integers free of large prime factors, Quart. J. Math. Oxford (2) $\mathbf{5 0}$ (1999), 111-130.

[13] H. Smida, Sur les puissances de convolution de la fonction de Dickman, Acta Arith. 59 $\mathrm{n}^{\circ} 2$ (1991), 124-143.

[14] H. Smida, Valeur moyenne des fonctions de Piltz sur les entiers sans grand facteur premier, Acta Arith. 63 (1993), 21-50.

[15] G. Tenenbaum, Sur une question d'Erdős et Schinzel, in :A tribute to Paul Erdös, 405443, Cambridge Univ. Press, Cambridge, 1990.

[16] G. Tenenbaum, Introduction à la théorie analytique et probabiliste des nombres, Cours spécialisés, $\mathrm{n}^{\circ} 1$, Société Mathématique de France (1995), xv + 457 pp.

[17] G. Tenenbaum, Crible d'Ératosthène et modèle de Kubilius, in : K. Győry, H. Iwaniec, J. Urbanowicz (eds.), Number Theory in Progress, Proceedings of the conference in honor of Andrzej Schinzel, Zakopane, Poland 1997, 1099-1129, Walter de Gruyter, Berlin, New York, 1999.

[18] G. Tenenbaum, Note on a paper by Joung Min Song, Acta Arith. 97 (2001) n4, 353-360.

[19] G. Tenenbaum, en collaboration avec J. Wu, Exercices corrigés de théorie analytique et probabiliste des nombres, Cours spécialisés, no. 2, Société Mathématique de France (1996), xiv + 251 pp.

[20] G. Tenenbaum \& J. Wu, Moyennes de certaines fonctions multiplicatives sur les entiers friables, J. reine angew. Math. 564 (2003), 119-166.

Guillaume Hanrot

INRIA Lorrain

Technopôle de Nancy-Brabois

615 , rue du Jardin Botanique

54602 Villers-lès-Nancy Cedex

France

Guillaume.Hanrot@loria.fr
Gérald Tenenbaum \& Jie Wu Institut Élie Cartan Université Henri Poincaré-Nancy 1 BP 239

54506 Vandœuvre Cedex

France

gerald.tenenbaum@iecn.u-nancy.fr wujie@iecn.u-nancy.fr 